\title{
Stabilizing effects of chromaticity and synchrotron emission on coupled-bunch transverse dynamics in storage rings
}

\author{
Ryan R. Lindberg๑* \\ Advanced Photon Source, Argonne National Laboratory, Argonne, Illinois 60439, USA
}

(Received 17 September 2020; accepted 29 January 2021; published 26 February 2021)

\begin{abstract}
We present a theory that can compute the transverse coupled-bunch instability growth rates at any chromaticity and for any longitudinal potential provided only that the long-range wakefield varies slowly over the bunch. The theory is expressed in terms of the usual coupled-bunch eigenvalues at zero chromaticity, and when the longitudinal motion is simple harmonic our solution only requires numerical root-finding that is easy to implement and fast to solve; the more general case requires some additional calculations, but is still relatively fast. The theory predicts that the coupled-bunch growth rates can be significantly reduced when the chromatic betatron tune spread is larger than the coupled-bunch growth rate at zero chromaticity. Our theoretical results are compared favorably with tracking simulations for the long-range resistive wall instability, and we also indicate how damping and diffusion from sychrotron emission can further reduce or even stabilize the dynamics.
\end{abstract}

DOI: 10.1103/PhysRevAccelBeams.24.024402

\section{INTRODUCTION}

Coupled-bunch transverse instabilities occur when longrange forces between particle bunches resonantly drive betatron oscillations. In a storage ring this results in a coordinated bunch instability whose growth rate increases with the total stored current [1,2]. The resulting coupledbunch motion can lead to emittance growth and even particle loss, so that predicting the growth rates is an important part of storage ring design.

The simplest theory of coupled-bunch motion models each bunch as a point particle, and the problem reduces to a collection of coupled oscillators $[3,4]$. The coupling matrix is specified by the long-range wakefield, and stability is determined by the eigenvalues of the linear system. The point bunch model can be derived from the coupled set of Vlasov equations in the limit that the long-range wakefield is essentially constant over the bunch (which is typically true) and that chromatic effects can be neglected (which often does not hold).

Theoretical treatments that apply at nonzero chromaticity must also include dynamics within each bunch, which is typically achieved by expressing each particle distribution function as a sum over orthogonal modes. For example, the formalism developed by Sacherer [5-7] expands each

\footnotetext{
*lindberg@aps.anl.gov

Published by the American Physical Society under the terms of the Creative Commons Attribution 4.0 International license. Further distribution of this work must maintain attribution to the author(s) and the published article's title, journal citation, and DOI.
}

bunch in terms of azimuthal and radial modes, which are in turn coupled together by the long-range wakefield so that the resulting matrix equation can become quite large and complicated. Typically used "handbook formulas" neglect the coupling between modes [8], but this becomes a poor approximation as the chromaticity increases. Laclare's approach [9], in which the distribution function is only azimuthally expanded into synchrotron modes and the problem reduces to an eigenvalue equation when mode coupling is neglected, provides better results at large chromaticity [10]. Nevertheless, these methods have a few deficiencies: they can become complicated and somewhat opaque when the instability growth rate becomes comparable to the synchrotron frequency, they typically neglect the damping and diffusion due to synchrotron emission, and they furthermore do not directly apply when the longitudinal motion is not simple harmonic.

This paper plans to address several of the aforementioned theoretical shortcomings, and compare the resulting predictions with detailed tracking simulations using the code ELEGANT [11]. While we could consider achieving many of these goals by applying the Vlasov-Fokker-Planck analysis developed in Refs. [12-14], we believe that more insight can be obtained by starting with a Vlasov-based approach similar to those used in Refs. [15-17], from which we obtain a dispersion relation that is identical to that of Ref. [18] when the potential is harmonic. We then include the physics of synchrotron emission, showing in detail how its diffusion can stabilize the dynamics at high chromaticity beyond that predicted by the usual radiation damping.

We begin in Sec. II by deriving the theory, with II A giving a brief overview of our approach and assumptions, 
Secs. II B-II D providing the details, and Sec. II E concluding with the dispersion-type integral solution that will form the basis of the rest of the paper. In Sec. III we apply the theory to a simple harmonic longitudinal potential appropriate for a single rf system, with the end result being the dispersion derived in Ref. [18] using a different approach. We show that the theory predicts two distinct regimes depending upon whether the zero-chromaticity growth rate is smaller or larger than the chromatic tune spread over the bunch; in the former "weak" limit the instability is strongly suppressed by the chromaticity, while in the latter "strong" instability regime this no longer holds.

Our theory differs from the classical one of Sacherer [5-7] in that it does not expand the perturbation into orthogonal modes; rather, growing solutions to the dispersion relation give all unstable modes. In the "weak" instability regime we find that the unstable solutions are approximately synchrotron modes that can be identified with a single value of the azimuthal index, and that there is only one such unstable mode per azimuthal number. Hence, we conclude that at nonzero chromaticity the instability is a superposition of several radial modes, and the often-used approximation that the radial modes remain uncoupled fails. As stated previously this result agrees with the findings of Ref. [10], and we will further discuss the connections between our theory and that of Sacherer and that of Laclare [9] in Sec. III C. Finally, our theory neglects the nonlinear tune-shift with amplitude, which applies if the emittance is sufficiently small such that the tune-shift over the bunch due to the transverse amplitude is much smaller than that due to chromaticity.

In Sec. III D we compare our theoretical predictions to those obtained from ELEGANT tracking simulations, finding very good agreement between the two if synchrotron emission can be neglected; when synchrotron emission is included the theory and simulation agree when the instability is strong, while in the weak limit the diffusion due to synchrotron emission further reduces the growth rate. We proceed in Sec. IIIE to derive an approximate dispersion relation that generalizes the Vlasov result by including longitudinal damping, and show how this can explain stability in the presence of synchrotron emission.

Next, in Sec. IV we apply the theory to a quartic longitudinal potential relevant to double rf systems tuned to "optimally" stretch the bunch. We show that the coupledbunch instability in a quartic potential has the same general phenomenology as that of a quadratic potential with the same bunch length: there is a weak regime in which the growth rate is suppressed somewhat more than that in a quadratic potential, and a strong regime where this reduction no longer holds. The theory agrees quite well with tracking simulations presented in Sec. IV B, and again indicates that the diffusion due to synchrotron emission can play an important role to stabilize the weak regime. Finally, we conclude in Sec. V.

\section{THEORY}

In this section we derive a theory that describes the stability of coupled-bunch transverse oscillations including chromaticity and for an arbitrary longitudinal potential. The derivation is rather long, so in Sec. II A we take a step back to sketch the major steps and assumptions involved. We then proceed in the next three subsections to carry out the program; those focused on results could skip this. The final product is an integral equation for the complex coupledbunch growth rate $\Omega$ in terms of the zero chromaticity growth rate $\lambda$, the characteristic synchrotron frequency $\alpha_{c} \sigma_{\delta} / \sigma_{t}$, and the head-tail (chromatic) phase shift over the bunch $k_{\xi} \sigma_{z}$; here, $\alpha_{c}$ is the momentum compaction, $\sigma_{\delta}$ is the rms energy spread, $\sigma_{z}=c \sigma_{t}$ is the rms bunch length, and $c k_{\xi}=\xi \omega_{0} / \alpha_{c}$ is the head-tail (chromatic) frequency for a ring with chromaticity $\xi$ and revolution frequency $\omega_{0}$.

\section{A. Outline of the derivation and assumptions}

Our starting point is the single particle dynamics including the (linear) transverse betatron motion, the longitudinal focusing, and the chromatic coupling between the two. Our first step is to choose a new set of coordinates that approximately eliminates the chromatic coupling; this coordinate change involves the well-known head-tail (or chromatic) phase, which will become one of the important parameters in the theory. Once we have two independent degrees of freedom, we then add the long-range transverse wakefield and simplify the problem by neglecting any change in the wakefield over the length of the bunch. This approximation is equivalent to assuming that all particles within a bunch receive the same wakefield kick, and that the dipole wakefield produced by any bunch is proportional to the transverse displacement its centroid; it is typically a very good approximation for the long-range resistive wall, and is often suitable for wakefields due to higher-order $\mathrm{rf}$ cavity modes.

Having specified the particle motion via the linear, chromatic, and long-range wakefield terms, we then tackle the problem of coupled-bunch stability using the Vlasov formalism. In this approach the distribution function of each bunch satisfies its own Vlasov equation, which in turn is coupled to the other bunches through the long-range transverse wakefield. Hence, the theory is Hamiltonian, and any dissipative effects of, e.g., synchrotron emission, can only be approximately included at the end. We then proceed to reduce the set of Vlasov equations into a coupled set of linear, ordinary differential equations for the transverse centroid positions. This process is basically done in two steps. The first step involves linearizing the problem about the equilibrium distribution function, multiplying by the transverse complex dipole displacement, and then integrating over the transverse degree of freedom. After dropping fast oscillating terms and then taking the Fourier transform, we are left with a coupled set of linear 
equations for the dipole-weighted longitudinal distribution function. The second step involves transforming to longitudinal action-angle variables, solving for the dipole-weighted longitudinal distribution function, and then integrating over longitudinal phase space to obtain a set of coupled equations for the transverse dipole moments in terms of the long-range wakefield and equilibrium quantities. Finally, we show how to reduce this to a set of independent equations through the usual matrix diagonalization, provided that the background equilibrium is the same for all bunches. This final requirement can be somewhat restrictive, but in the Appendix A we also indicate how it can be relaxed to include periodic variations in the equilibrium that may arise due to beam-loading of rf cavities with a nonuniform fill pattern. At the end, we will find an integral equation for the complex coupled-bunch growth rate $\Omega$ in terms of the zero chromaticity growth rate $\lambda$ and the equilibrium distribution.

\section{B. Particle equations of motion}

We begin with the single particle Hamiltonian describing motion in a linear lattice including chromatic effects; we will add the long-range wakefield shortly, and then the dissipative effects of synchrotron emission after that. We use the position $s$ along the ring as the independent variable and adopt the coordinates $\left(z, p_{z}\right)$ to describe the longitudinal motion. These are a canonical coordinate pair, with $z=s-c t$ denoting the comoving position of a relativistic particle moving at (approximately) the speed of light $c$ that arrives at location $s$ at time $t$, while $p_{z}=-\left(\gamma-\gamma_{r}\right) / \gamma_{r}$ gives the (negative) scaled energy deviation of a particle from the reference energy $\gamma_{r}$. In the transverse plane we introduce the dynamical angleaction variables $(\Psi, \mathcal{J})$, which characterize the phase and amplitude of the betatron oscillation and are related to the transverse position via $y=\sqrt{2 \beta_{y} \mathcal{J}} \cos \Psi$. In terms of these variables, the linear plus chromatic motion over one turn can be described by the ring-averaged (or smooth) Hamiltonian

$$
\begin{aligned}
\mathcal{H}_{0} & =\mathcal{H}_{z}\left(z, p_{z}\right)+\frac{\omega_{\beta}}{c} \mathcal{J}-\frac{\omega_{0} \xi}{c} p_{z} \mathcal{J}[1+\cos (2 \Psi)] \\
& \approx \mathcal{H}_{z}\left(z, p_{z}\right)+\frac{\omega_{\beta}}{c} \mathcal{J}-\frac{\omega_{0} \xi}{c} p_{z} \mathcal{J} .
\end{aligned}
$$

In the second line we have dropped the term $\sim \cos (2 \Psi)$ under the assumption that its effect on the betatron motion averages to zero. Then, we find that the linear dynamics preserves the action $\mathcal{J}$ as an invariant, while the angle $\Psi$ increases by the energy-dependent (chromatic) betatron phase $\left(\omega_{\beta}-\xi \omega_{0} p_{z}\right) T_{0}$ every turn, where $\omega_{\beta}$ is the betatron frequency, $\xi$ is the chromaticity, and $T_{0}=2 \pi / \omega_{0}$ is the revolution time. In addition, we will assume that the evolution associated with the longitudinal Hamiltonian
$\mathcal{H}_{z}\left(z, p_{z}\right)$ occurs over a timescale much longer than that of the transverse betatron motion, but otherwise leave $\mathcal{H}_{z}$ to be specified later.

The dynamics in the transverse plane is only independent from that in the longitudinal plane when the chromaticity vanishes; to decouple the motion when $\xi \neq 0$ we transform coordinates using the "type-two" $[19,20]$ mixed-variable generating function given by

$$
S_{2}\left(\bar{p}_{z}, \overline{\mathcal{J}}, z, \Psi\right)=\overline{\mathcal{J}} \Psi+\bar{p}_{z} z+\frac{\omega_{0} \xi}{\alpha_{c} c} \overline{\mathcal{J}} z
$$

where bars indicate the new coordinates. The new and old variables are related by

$$
\begin{aligned}
& \bar{z}=\frac{\partial S_{2}}{\partial \bar{p}_{z}}=z \quad p_{z}=\frac{\partial S_{2}}{\partial z}=\bar{p}_{z}+\frac{\omega_{0} \xi}{\alpha_{c} c} \overline{\mathcal{J}} \\
& \bar{\Psi}=\frac{\partial S_{2}}{\partial \overline{\mathcal{J}}}=\Psi+\frac{\omega_{0} \xi}{\alpha_{c} c} z \quad \mathcal{J}=\frac{\partial S_{2}}{\partial \Psi}=\overline{\mathcal{J}}
\end{aligned}
$$

and we find that inserting these into $\mathcal{H}_{0}$ eliminates the chromatic coupling term $\left(\omega_{0} \xi / c\right) p_{z} \mathcal{J}$. Furthermore, since the energy spread $\sigma_{\delta}$ is typically several orders of magnitude larger than $\left(\omega_{0} \xi / \alpha_{c} c\right) \varepsilon_{y}$, we can approximately set $\bar{p}_{z}=p_{z}$ in $\mathcal{H}_{z}\left(z, p_{z}\right)$, and $S_{2}$ generates a coordinate transformation that decouples transverse and longitudinal coordinates. Finally, we find that the new betatron angle is related to the old one by adding on the so-called "head-tail phase" [21,22]

$$
\frac{\omega_{0} \xi}{\alpha_{c} c} \bar{z}=\frac{2 \pi \xi}{\alpha_{c} c T_{0}} z \equiv k_{\xi} z
$$

The head-tail phase arises because the betatron frequency depends linearly on the energy for $\xi \neq 0$, which in turn leads to the betatron phase accumulating a shift that is proportional to the longitudinal coordinate $z$ as it performs synchrotron oscillations.

In summary, at lowest order the chromatic (head-tail) coordinate transformation eliminates the chromatic part of $\mathcal{H}_{0}$ while replacing the betatron phase via $\Psi \rightarrow \bar{\Psi}-k_{\xi} \bar{z}$. The other new coordinates are essentially the same as the old ones, so for simplicity we will only retain the bar on the new betatron phase; the new and uncoupled Hamiltonian is then

$$
\mathcal{H}_{0} \approx \mathcal{H}_{z}\left(z, p_{z}\right)+\frac{\omega_{\beta}}{c} \mathcal{J}
$$

\section{Wakefields in the Vlasov description}

Now that we have the nicely uncoupled, chromatic Hamiltonian (6), it is time to add the perturbing transverse wakefields. To do this, we introduce the particle distribution function of bunch $j$ as $F_{j}(\mathcal{Z} ; s)$, where we abbreviate 
the phase-space coordinates as $\mathcal{Z}=\left(z, p_{z}, \Psi, \mathcal{J}\right)$, and normalize $F_{j}$ such that $\int d \mathcal{Z} F_{j}=1$ for $0 \leq j \leq N_{b}-1$, with $N_{b}$ the number of bunches. Furthermore, we will include both the dipolar wakefield, for which the kick on the trailing particle is proportional to the displacement of the leading particle, and the quadrupolar wakefield that comes about when the vacuum chamber is not cylindrically symmetric and whose effect on the trailing particle scales with its own displacement. The total kick on any particle is obtained by summing both wakefield contributions over all bunches in the ring and over all previous turns. We define the equilibrium centroid spacing between bunch $n$ and $j$ to be $L_{n, j}$ with $L_{n, j}>0$ if $j>n$ and $L_{n, j}=-L_{n, j}$ if $j \leq n$, in which case we can write the potential due to the quadrupolar wakefield as

$$
\begin{aligned}
\mathcal{V}_{Q} & =\frac{y^{2}}{2} \sum_{j=0}^{N_{b}-1} \frac{e^{2} N_{j}}{\gamma m c T_{0}} \sum_{\ell=0} \int d \mathcal{Z}^{\prime} F_{j}\left(\mathcal{Z}^{\prime} ; s-\ell c T_{0}\right) W_{Q}\left[z-\left(z^{\prime}+\ell c T_{0}+L_{n, j}\right)\right] \\
& \approx \mathcal{J} \sum_{j=0}^{N_{b}-1} \frac{e^{2} N_{j}}{2 \gamma m c T_{0}} \sum_{\ell=0}^{\infty} \int d \mathcal{Z}^{\prime} F_{j}\left(\mathcal{Z}^{\prime} ; s-\ell c T_{0}\right) W_{Q}^{\beta}\left[z-\left(z^{\prime}+\ell c T_{0}+L_{n, j}\right)\right],
\end{aligned}
$$

where we used $y^{2}=2 \beta_{y} \mathcal{J} \cos ^{2} \bar{\Psi} \approx \beta_{y} \mathcal{J}$ under the assumption that we can neglect fast oscillations at twice the betatron frequency $\sim \cos (2 \bar{\Psi})$, and have defined the beta-function-weighted wakefield $W_{Q}^{\beta}(\zeta)=\beta_{y} W_{Q}(\zeta)$. Our final simplification of the long-range wakefield will be to assume that it varies slowly over distances of order the bunch length $\sigma_{z}$; we therefore Taylor expand the long-range wakefields about the equilibrium bunch position that has $z-z^{\prime}=0$ and keep only the lowest order term. In this case (7) reduces to

$$
\mathcal{V}_{Q} \approx \mathcal{J} \sum_{j=0}^{N_{b}-1} \frac{e^{2} N_{j}}{2 \gamma m c T_{0}} \sum_{\ell=0}^{\infty} W_{Q}^{\beta}\left(-\ell c T_{0}-L_{n, j}\right) \int d \mathcal{Z}^{\prime} F_{j}\left(\mathcal{Z}^{\prime} ; s-\ell c T_{0}\right)=\mathcal{J} \frac{\omega_{Q}}{c},
$$

and we find that the effect of the long-range quadrupolar wakefield is to shift the betatron frequency by an amount $\omega_{Q}$ that is independent of $s$ (see, e.g., [23]).

For the dipolar wakefield, we perform a similar Taylor expansion and change of coordinates, finding that

$$
\begin{aligned}
\mathcal{V}_{D} & =y \sum_{j=0}^{N_{b}-1} \frac{e^{2} N_{j}}{\gamma m c T_{0}} \sum_{\ell=0}^{\infty} \int d \mathcal{Z}^{\prime} y^{\prime} F_{j}\left(\mathcal{Z}^{\prime} ; s-\ell c T_{0}\right) W_{D}\left[z-\left(z^{\prime}+\ell c T_{0}+L_{n, j}\right)\right] \\
& \approx \sqrt{\mathcal{J}} \cos \left(\bar{\Psi}-k_{\xi} z\right) \sum_{j=0}^{N_{b}-1} \frac{2 e^{2} N_{j}}{\gamma m c T_{0}} \sum_{\ell=0}^{\infty} W_{D}^{\beta}\left(-\ell c T_{0}-L_{n, j}\right) \int d \mathcal{Z}^{\prime} \sqrt{\mathcal{J}^{\prime}} \cos \left(\bar{\Psi}^{\prime}-k_{\xi} z^{\prime}\right) F_{j}\left(\mathcal{Z}^{\prime} ; s-\ell c T_{0}\right) \\
& =\sqrt{\mathcal{J}} \cos \left(\bar{\Psi}-k_{\xi} z\right) \sum_{j=0}^{N_{b}-1} \mathcal{W}_{n, j}(s),
\end{aligned}
$$

where for notational brevity we have defined $\mathcal{W}_{n, j}$ to be proportional to the kick that a particle in bunch $n$ receives due to the centroid displacement of bunch $j$.

The final step of our treatment of the single particle Hamiltonian involves transforming the longitudinal coordinates to the angle-action pair $(\Phi, \mathcal{I})$ of the unperturbed, longitudinal $\mathcal{H}_{z}\left(z, p_{z}\right)$. While the formulas defining $\left(z, p_{z}\right) \rightarrow(\Phi, \mathcal{I})$ will depend upon the longitudinal potential, in general the transformation results in $\mathcal{H}_{z}\left(z, p_{z}\right) \rightarrow \mathcal{H}_{z}(\mathcal{I})$, so that inserting Eqs. (6), (8), and (10) into the single particle Hamiltonian $\mathcal{H}=\mathcal{H}_{0}+\mathcal{V}_{Q}+\mathcal{V}_{D}$ yields

$$
\mathcal{H}(\mathcal{Z} ; s)=\mathcal{H}_{z}(\mathcal{I})+\frac{\omega_{\beta}+\omega_{Q}}{c} \mathcal{J}+\sqrt{\mathcal{J}} \cos \left[\bar{\Psi}-k_{\xi} z(\Phi, \mathcal{I})\right] \sum_{j=0}^{N_{b}-1} \mathcal{W}_{n, j}(s),
$$

where, again, $\mathcal{W}_{n, j}$ is the dipolar kick defined by (9)-(10), and $z(\Phi, \mathcal{I})$ depends on the rf potential that will be specified later. 


\section{Coupled-bunch equations including synchrotron emission}

The Hamiltonian (11) specifies the conservative equations of motion within our model, and to determine collective stability we will consider the Vlasov-Fokker-Planck equations associated with $\mathcal{H}$. We assume that the number of electrons in each bunch is constant, so that each bunch $n$ satisfies its own Vlasov-Fokker-Planck equation given by

$$
\frac{\partial F_{n}}{\partial s}+\frac{\omega(\mathcal{I})}{c} \frac{\partial F_{n}}{\partial \Phi}+\frac{\omega_{\beta}+\omega_{Q}}{c} \frac{\partial F_{n}}{\partial \Psi}+\sum_{j=0}^{N_{b}-1} \mathcal{W}_{n, j}\left[\frac{\cos \left(\Psi-k_{\xi} z\right)}{2 \sqrt{\mathcal{J}}} \frac{\partial F_{n}}{\partial \Psi}+\sqrt{\mathcal{J}} \sin \left(\Psi-k_{\xi} z\right) \frac{\partial F_{n}}{\partial \mathcal{J}}\right]=\mathcal{E}_{\mathrm{FP}}\left[F_{n}\right]
$$

where we have introduced the amplitude-dependent longitudinal (i.e., synchrotron) frequency $\omega(\mathcal{I})=\partial \mathcal{H}_{z} / \partial \mathcal{I}$, we have neglected the (assumed small) transverse wakefield in the longitudinal force, and the dissipative effects of synchrotron emission are incorporated with the Fokker-Planck operator $\mathcal{E}_{\mathrm{FP}}$. In particular, the emission of synchrotron radiation results in damping and diffusion that we model with the following differential operator (see, e.g., [24,25])

$$
\mathcal{E}_{\mathrm{FP}}=\frac{2}{c \tau_{z}}\left[\sigma_{\delta}^{2} \frac{\partial^{2}}{\partial p_{z}^{2}}+p_{z} \frac{\partial}{\partial p_{z}}+1\right]+\frac{2}{c \tau_{y}}\left[\varepsilon_{y, 0}\left(\mathcal{J} \frac{\partial^{2}}{\partial \mathcal{J}^{2}}+\frac{1}{4 \mathcal{J}} \frac{\partial^{2}}{\partial \Psi^{2}}+\frac{\partial}{\partial \mathcal{J}}\right)+\left(\mathcal{J} \frac{\partial}{\partial \mathcal{J}}+1\right)\right],
$$

where $\tau_{z}$ and $\tau_{y}$ are the longitudinal and vertical damping times, respectively, $\sigma_{\delta}$ is the equilibrium energy spread, and $\varepsilon_{y, 0}$ is the equilibrium emittance.

To get an equation for the transverse dipole moment, we multiply (12) by $\sqrt{\mathcal{J}} e^{-i \Psi}$ and integrate over transverse phase space. We use

$$
\sqrt{\mathcal{J}} e^{-i \Psi} \frac{\partial F}{\partial \Psi}=\frac{\partial}{\partial \Psi}\left(\sqrt{\mathcal{J}} e^{-i \Psi} F\right)+i \sqrt{\mathcal{J}} e^{-i \Psi} F, \quad \mathcal{J} e^{-i \Psi} \frac{\partial F}{\partial \mathcal{J}}=\frac{\partial}{\partial \mathcal{J}}\left(\mathcal{J} e^{-i \Psi} F\right)-e^{-i \Psi} F,
$$

to simplify the Hamiltonian left-hand side of (12), and evaluate the right-hand side $\mathcal{E}_{\mathrm{FP}}\left[F_{n}\right]$ using

$$
\sqrt{\mathcal{J}} e^{-i \Psi}\left(\mathcal{J} \frac{\partial^{2} F_{n}}{\partial \mathcal{J}^{2}}+\frac{1}{4 \mathcal{J}} \frac{\partial^{2} F_{n}}{\partial \Psi^{2}}+\frac{\partial F_{n}}{\partial \mathcal{J}}\right)=\frac{\partial}{\partial \mathcal{J}}\left[\left(\mathcal{J}^{3 / 2} \frac{\partial}{\partial \mathcal{J}}-\sqrt{\mathcal{J}}\right) e^{-i \Psi} F_{n}\right]+\frac{\partial}{\partial \Psi}\left[\frac{e^{-i \Psi}}{4 \sqrt{\mathcal{J}}}\left(\frac{\partial}{\partial \Psi}+i\right) F_{n}\right]
$$

and

$$
\sqrt{\mathcal{J}} e^{-i \Psi}\left(\mathcal{J} \frac{\partial F_{n}}{\partial \mathcal{J}}+F_{n}\right)=-\frac{1}{2} \sqrt{\mathcal{J}} e^{-i \Psi} F_{n}+\frac{\partial}{\partial \mathcal{J}}\left(\mathcal{J}^{3 / 2} e^{-i \Psi} F_{n}\right)
$$

Then, all the transverse dependence involves either terms $\propto \sqrt{\mathcal{J}} e^{-i \Psi} F_{n}$ or are derivatives with respect to $\Psi$ or $\mathcal{J}$ that vanish upon integration over transverse phase space. Hence, if we define $f_{n}\left(z, p_{z} ; s\right)=\int d \mathcal{J} d \Psi \sqrt{\mathcal{J}} e^{-i \Psi} F_{n}(\mathcal{Z} ; s)$ and integrate over transverse phase space we arrive at

$$
\begin{aligned}
& {\left[\frac{\partial}{\partial s}+\frac{\omega(\mathcal{I})}{c} \frac{\partial}{\partial \Phi}+i \frac{\omega_{\beta}+\omega_{Q}}{c}+\frac{1}{c \tau_{y}}\right] f_{n}(\Phi, \mathcal{I} ; s)} \\
& \quad+f_{n}(\Phi, \mathcal{I} ; s) e^{-i k_{\xi} z} \sum_{j=0}^{N_{b}-1} \frac{i e^{2} N_{e, j}}{2 \gamma m c^{3} T_{0}} \sum_{\ell=0}^{\infty} W_{D}\left(-\ell c T_{0}-L_{n j}\right) \int d \Phi^{\prime} d \mathcal{I}^{\prime}\left[f_{j}\left(\Phi^{\prime}, \mathcal{I}^{\prime} ; s-\ell c T_{0}\right) e^{i k_{\xi} z^{\prime}}+\text { c.c. }\right]=\mathcal{E}_{\mathrm{FP}}^{\|}\left[f_{n}\right]
\end{aligned}
$$

Here, we have used the definition in Eqs. (9)-(10) to expand $\mathcal{W}_{n, j}$ in terms of the wakefield and the longitudinal distribution. The first line of (17) describes the single-bunch evolution of the centroid-weighted longitudinal distribution, and includes the synchrotron and betatron oscillations as well as the radiation damping that stabilizes the vertical oscillations. The second line contains the long-range wakefield perturbation from all other bunches, along with the reduced, longitudinal Fokker-Planck operator defined by

$$
\mathcal{E}_{\mathrm{FP}}^{\|}\left[f_{n}\right]=\frac{2}{c \tau_{z}}\left(\sigma_{\delta}^{2} \frac{\partial^{2}}{\partial p_{z}^{2}}+p_{z} \frac{\partial}{\partial p_{z}}+1\right) f_{n}(\Phi, \mathcal{I} ; s) .
$$


Now, we linearize the problem by separating $f_{n}$ into its equilibrium part $\bar{f}_{n}$ and its perturbation $g_{n}$ via

$$
\begin{aligned}
f_{n}(\Phi, \mathcal{I} ; s) & =\bar{f}_{n}(\mathcal{I})+e^{-i\left(\omega_{\beta}+\omega_{Q}\right) s} g_{n}(\Phi, \mathcal{I} ; s) \\
& =\bar{f}_{n}(\mathcal{I})+e^{-i\left(\omega_{\beta}+\omega_{Q}\right) s} \int d \Omega e^{-i \Omega s / c} \tilde{g}_{n}(\Phi, \mathcal{I} ; \Omega),
\end{aligned}
$$

where the final equality introduces the Fourier transform of the perturbation $\tilde{g}_{n}$. Note that we have extracted the linear betatron motion with the factor $e^{-i\left(\omega_{\beta}+\omega_{Q}\right) s}$, so that in the absence of wakefields $\Omega=0$. Inserting the expansion (19) into (17), multiplying by $e^{i\left(\omega_{\beta}+\omega_{Q}\right) s}$, and taking the Fourier transform of the result leads to

$$
\begin{aligned}
& {\left[-\frac{i \Omega}{c}+\frac{1}{c \tau_{y}}+\frac{\omega(\mathcal{I})}{c} \frac{\partial}{\partial \Phi}\right] \tilde{g}_{n}(\Phi, \mathcal{I} ; \Omega)+\sum_{j=0}^{N_{b}-1} \frac{i e^{2} N_{e, j}}{2 \gamma m c^{3} T_{0}} \sum_{\ell=0}^{\infty} W_{D}\left(-\ell c T_{0}-L_{n j}\right) e^{i \ell\left(\omega_{\beta}+\omega_{Q}+\Omega\right) T_{0}}} \\
& \times \bar{f}_{n}(\mathcal{I}) e^{-i k_{\xi} z} \int d \Phi^{\prime} d \mathcal{I}^{\prime}\left[\tilde{g}_{j}\left(\Phi^{\prime}, \mathcal{I}^{\prime} ; \Omega\right) e^{i k_{\xi} z^{\prime}}+\tilde{g}_{j}^{*}\left(\Phi^{\prime}, \mathcal{I}^{\prime} ;-\Omega-2 \omega_{\beta}-2 \omega_{Q}\right) e^{i k_{\xi} z^{\prime}}\right]=\mathcal{E}_{\mathrm{FP}}^{\|}\left[\tilde{g}_{n}\right] .
\end{aligned}
$$

We will assume that the perturbation is weak in the sense that any modification to the betatron frequency is much smaller than the revolution frequency, $|\Omega| \ll 1 / T_{0}$. In this case we use $e^{i \ell\left(\omega_{\beta}+\omega_{Q}+\Omega\right) T_{0}} \approx e^{i \ell\left(\omega_{\beta}+\omega_{Q}\right) T_{0}}$ in the top line of (20), and additionally use the fact that $\tilde{g}$ is centered about $\Omega \approx 0$ to drop the second term in the bottom line. Then, we see that the long-range wakefield term splits into a product of three pieces: the first depends upon the perturbation $\tilde{g}_{n}$ and will become our dynamical variable, the second contains the coupling force due to wakefields, and the third is a function of the single-bunch equilibrium. We will denote the first two of these as

$$
\begin{aligned}
\mathcal{D}_{j}(\Omega) & \equiv \int d \Phi d \mathcal{I} \tilde{g}_{j}(\Phi, \mathcal{I} ; \Omega) e^{i k_{\xi} z}, \\
\mathrm{M}_{n, j} & =\frac{e^{2} N_{e, j}}{2 \gamma m c^{2} T_{0}} \sum_{\ell=0}^{\infty} W_{D}\left(-\ell c T_{0}-L_{n j}\right) e^{i \ell\left(\omega_{\beta}+\omega_{Q}\right) T_{0}},
\end{aligned}
$$

so that $\mathcal{D}_{j}(\Omega)$ is the (chromatic) dipole moment of the $j$ th bunch, while $\mathrm{M}$ is the chromaticity-independent coupling matrix due to the long-range wakefields. The matrix $M$ is essentially the same as that used in the point-bunch model of Thompson and Ruth [4], which in turn can be related to the frequency/impedance theory of, e.g., Ref. [6]. Using the definitions (21), we find that our stability equation reduces to

$$
\begin{aligned}
& {\left[\Omega+\frac{i}{\tau_{y}}+i \omega(\mathcal{I}) \frac{\partial}{\partial \Phi}\right] \tilde{g}_{n}(\Phi, \mathcal{I} ; \Omega)-\bar{f}_{n}(\mathcal{I}) e^{-i k_{\xi} z(\Phi, \mathcal{I})}} \\
& \quad \times \sum_{j=0}^{N_{b}-1} \mathrm{M}_{n, j} \mathcal{D}_{j}(\Omega)=i c \mathcal{E}_{\mathrm{FP}}^{\|}\left[\tilde{g}_{n}\right]
\end{aligned}
$$

Solving the full Fokker-Planck equation (22) requires knowing the longitudinal potential and equilibrium, and we will return to that for a simple harmonic potential in Sec. III E. In the next section we instead take the Vlasov (dissipation-free) limit by setting $\mathcal{E}_{\mathrm{FP}}^{\|}[\tilde{g}] \rightarrow 0$, and show that in this case we can derive a relatively simple dispersion relation for the coupled bunch problem.

\section{E. Coupled-bunch dispersion relation in the Vlasov (no damping or diffusion) limit}

In this section we assume that the effects of synchrotron damping and diffusion are negligible. The validity of this limit is complicated by the fact that the diffusive part of $\mathcal{E}_{\mathrm{FP}}^{\|}$ involves second derivatives with respect to $p_{z}$, and therefore will depend upon the phase space density profile of the unstable perturbation. We will revisit how the applicability of our Vlasov results depends upon the damping, chromaticity, and strength of the wakefields in Sec. III E, but for now we just set $\mathcal{E}_{\mathrm{FP}}^{\|}[\tilde{g}] \rightarrow 0$ and see where that takes us. Under this assumption we can reduce Eq. (22) into an equation for the transverse dipole moment $\mathcal{D}(\Omega)$, thereby eliminating the dependence on the longitudinal variables $(\Phi, \mathcal{I})$. To do this, we use the approach of $[15,16]$ to rewrite (22) with vanishing right-hand side as follows:

$$
e^{i \Omega \Phi / \omega(\mathcal{I})} \frac{\partial}{\partial \Phi}\left[e^{-i \Omega \Phi / \omega(\mathcal{I})} \tilde{g}_{n}\right]=-\frac{i e^{2} \bar{f}_{n}(\mathcal{I}) e^{-i k_{\xi} z(\Phi, \mathcal{I})}}{2 \gamma m c^{2} T_{0} \omega(\mathcal{I})} \sum_{j=0}^{N_{b}-1} N_{e, j} \mathcal{D}_{j}(\Omega) \sum_{\ell=0}^{\infty} W_{D}\left(-\ell c T_{0}-L_{n j}\right) e^{i \ell\left(\omega_{\beta}+\omega_{Q}\right) T_{0}}
$$

Then, we can integrate over angle from $\Phi$ to $\Phi+2 \pi$ and use the fact that $\tilde{g}_{n}$ is periodic in $\Phi$ to obtain 
$\tilde{g}_{n}=\frac{e^{2} \bar{f}_{n}(\mathcal{I}) e^{2 \pi i \Omega}}{2 i \gamma m c^{2} T_{0} \omega(\mathcal{I})} \frac{e^{i \Omega \Phi / \omega(\mathcal{I})}}{1-e^{2 i \pi \Omega}} \int_{\Phi}^{\Phi+2 \pi} d \Phi^{\prime} e^{-i k_{\xi} z\left(\Phi^{\prime}, \mathcal{I}\right)} e^{-i \Omega \Phi^{\prime} / \omega(\mathcal{I})} \sum_{j=0}^{N_{b}-1} N_{e, j} \mathcal{D}_{j}(\Omega) \sum_{\ell=0}^{\infty} W_{D}\left(-\ell c T_{0}-L_{n j}\right) e^{i \ell\left(\omega_{\beta}+\omega_{Q}\right) T_{0}}$.

Finally, we obtain a closed-form coupled set of expressions for the dipole moments $\mathcal{D}_{n}(\Omega)$ by multiplying by $e^{i k_{\xi} z(\Phi, \mathcal{I})}$ and integrating over longitudinal phase space $(\Phi, \mathcal{I})$. We then simplify the resulting expression by changing coordinates to $\theta=\Phi-\Phi^{\prime}+2 \pi$, finding that

$$
\begin{aligned}
\mathcal{D}_{n}(\Omega)= & \sum_{j=0}^{N_{b}-1} \frac{e^{2} N_{e, j} \mathcal{D}_{j}(\Omega)}{2 i \gamma m c^{2} T_{0}} \sum_{\ell=0}^{\infty} W_{D} e^{i \ell\left(\omega_{\beta}+\omega_{Q}\right) T_{0}} \int_{0}^{\infty} d \mathcal{I} \frac{\bar{f}_{n}(\mathcal{I})}{\omega(\mathcal{I})} \int_{0}^{2 \pi} d \Phi \frac{e^{i \Omega \Phi / \omega} e^{i k_{\xi} z}}{e^{-2 \pi i \Omega}-1} \int_{\Phi}^{\Phi+2 \pi} d \Phi^{\prime} e^{-i k_{\xi} z^{\prime}} e^{-i \Omega \Phi^{\prime} / \omega} \\
= & \frac{e^{2}}{2 i \gamma m c^{2} T_{0}} \sum_{j=0}^{N_{b}-1} N_{e, j} \mathcal{D}_{j}(\Omega) \sum_{\ell=0}^{\infty} W_{D}\left(-\ell c T_{0}-L_{n j}\right) e^{i \ell\left(\omega_{\beta}+\omega_{Q}\right) T_{0}} \\
& \times \int_{0}^{\infty} d \mathcal{I} \frac{\bar{f}_{n}(\mathcal{I})}{\omega(\mathcal{I})} \int_{0}^{2 \pi} d \Phi \frac{e^{i k_{\xi} z(\Phi, \mathcal{I})}}{1-e^{2 \pi i \Omega}} \int_{0}^{2 \pi} d \theta e^{-i k_{\xi} z(\Phi-\theta, \mathcal{I})} e^{i \Omega \theta / \omega(\mathcal{I})} .
\end{aligned}
$$

To make further progress, we now assume that the equilibrium distribution $\bar{f}_{n}$ is independent of $n$, but we show in Appendix A how to generalize our approach to fill patterns with two or more approximately identical bunch trains. Assuming that $\bar{f}_{n}(\mathcal{I})=\bar{f}(\mathcal{I})$ for all $n$ reduces $(25)$ to the matrix problem

$$
\begin{aligned}
\mathcal{D}_{n}= & \sum_{j=0}^{N_{b}-1} \mathrm{M}_{n, j} \mathcal{D}_{j} \int_{0}^{\infty} d \mathcal{I} \int_{0}^{2 \pi} d \Phi \frac{\bar{f}(\mathcal{I})}{\omega(\mathcal{I})} \frac{e^{i k_{\xi} z(\Phi, \mathcal{I})}}{1-e^{2 \pi i \Omega / \omega(\mathcal{I})}} \\
& \times \int_{0}^{2 \pi} d \theta e^{i \Omega \theta / \omega(\mathcal{I})} e^{-i k_{\xi} z(\Phi-\theta, \mathcal{I})}
\end{aligned}
$$

where the coupling matrix $\mathrm{M}$ has components

$$
\mathrm{M}_{n, j}=\frac{e^{2} N_{e, j}}{2 \gamma m c^{2} T_{0}} \sum_{\ell=0}^{\infty} W_{D}\left(-\ell c T_{0}-L_{n j}\right) e^{i \ell\left(\omega_{\beta}+\omega_{Q}\right) T_{0}} .
$$

The matrix $\mathrm{M}$ is essentially the same as the theory of Thompson and Ruth [4], which in turn can be understood as the time-domain version of the familiar impedance-based theory of, e.g., [6]. The formula (26) is particularly convenient because it divides into two pieces: one piece that is the usual matrix theory, and the other which involves the longitudinal distribution function and the chromaticity. The former can be diagonalized in the standard way; if we assume that this has been done, i.e., a matrix $U$ has been found such that $\mathrm{UMU}^{-1}=\lambda \mathbb{I}$ with $\mathbb{I}$ the identity, then

$$
\begin{aligned}
1= & \frac{\lambda}{2 \pi i} \int_{0}^{\infty} d \mathcal{I} \int_{0}^{2 \pi} d \Phi \frac{2 \pi \bar{f}(\mathcal{I})}{\omega(\mathcal{I})} \frac{e^{i k_{\xi} z(\Phi, \mathcal{I})}}{1-e^{2 \pi i \Omega / \omega(\mathcal{I})}} \\
& \times \int_{0}^{2 \pi} d \theta e^{i \Omega \theta / \omega(\mathcal{I})} e^{-i k_{\xi} z(\Phi-\theta, \mathcal{I})}
\end{aligned}
$$

Now $z(\Phi, \mathcal{I})$ is periodic in $\Phi$, and can be expanded in a Fourier series. For the cases that we consider we can usefully approximate the motion using only the lowest harmonic in this series, in which case we can write $z(\Phi, \mathcal{I}) \approx \zeta(\mathcal{I}) \cos \Phi$, where $\zeta(\mathcal{I})$ is a function of action that will be determined by the longitudinal Hamiltonian $\mathcal{H}_{z}$. Then, we can take two different routes to simplify Eq. (28). One route, which we find useful for some numerical calculations and detail in Appendix $\mathrm{C}$, uses the Jacobi-Anger identity to integrate over angles. The second path will form the basis of our subsequent theoretical analysis, and combines the exponentials involving $z$ in $(28)$ as

$$
\begin{aligned}
e^{-i k_{\xi}[z(\Phi-\theta, \mathcal{I})-z(\Phi, \mathcal{I})]} & =e^{-i k_{\xi} \zeta(\mathcal{I})[\cos (\Phi-\theta)-\cos \Phi]} \\
& =e^{i k_{\xi} \zeta(\mathcal{I})[(1-\cos \theta) \cos \Phi-\sin \theta \sin \Phi]} \\
& =e^{2 i k_{\xi} \zeta(\mathcal{I}) \sin (\theta / 2) \cos (\Phi+\psi)},
\end{aligned}
$$

where $\psi$ is a phase that is irrelevant to our calculation. Inserting (29) into (28), we find that the integration over $\Phi$ can be performed in the following way

$$
\begin{aligned}
& \int_{0}^{2 \pi} d \Phi e^{-i k_{\xi}[z(\Phi-\theta, \mathcal{I})-z(\Phi, \mathcal{I})]} \\
& \quad=\int_{0}^{2 \pi} d \Phi e^{2 i k_{\xi} \zeta \sin (\theta / 2) \cos (\Phi+\psi)} \\
& \quad=\int_{0}^{2 \pi} d \Phi \sum_{\ell} i^{\ell} J_{\ell}\left[2 k_{\xi} \zeta(\mathcal{I}) \sin (\theta / 2)\right] e^{i \ell(\Phi+\psi)} \\
& \quad=2 \pi J_{0}\left[2 k_{\xi} \zeta(\mathcal{I}) \sin (\theta / 2)\right],
\end{aligned}
$$

where $J_{0}(x)$ is the usual Bessel function.

Finally, we will find it convenient to introduce the dimensionless eigenvalue $\hat{\lambda}$ and complex growth rate $\hat{\Omega}$ by scaling each with a characteristic value of the synchrotron frequency. We do this using $\alpha_{c} \sigma_{\delta} / \sigma_{t}$, which is of the order of the mean synchrotron frequency for a bunch of 
rms energy spread $\sigma_{\delta}$ and temporal duration $\sigma_{t}$, so that we introduce

$$
\hat{\lambda}=\frac{\lambda}{\alpha_{c} \sigma_{\delta} / \sigma_{t}}, \quad \hat{\Omega}=\frac{\Omega}{\alpha_{c} \sigma_{\delta} / \sigma_{t}} .
$$

Then, in terms of the scaled longitudinal frequency $\hat{\omega}=\omega(\mathcal{I}) /\left(\alpha_{c} \sigma_{\delta} / \sigma_{t}\right)$ stability is determined by

$$
\begin{aligned}
1= & -i \hat{\lambda} \int_{0}^{\infty} d \mathcal{I} \frac{2 \pi \bar{f}(\mathcal{I})}{\hat{\omega}(\mathcal{I})\left[1-e^{2 \pi i \hat{\Omega} / \hat{\omega}(\mathcal{I})}\right]} \\
& \times \int_{0}^{2 \pi} d \theta J_{0}\left[2 k_{\xi} \zeta(\mathcal{I}) \sin (\theta / 2)\right] e^{i \hat{\Omega} \theta / \hat{\omega}(\mathcal{I})} .
\end{aligned}
$$

Solving the integral equation (39) gives the (scaled) coupled bunch complex growth rate $\hat{\Omega}$ in terms of two dimensionless parameters: the scaled, $\xi=0$ matrix eigenvalue $\hat{\lambda}$, and the chromatic (head-tail) phase shift $k_{\xi} \sigma_{z}$. Equation (32) can in principal be solved once we have specified the longitudinal dynamics through $\mathcal{H}_{z}\left(z, p_{z}\right)$ and equilibrium $\bar{f}(\mathcal{I})$; the former determines the frequency $\omega(\mathcal{I})$ and Fourier coefficient $\zeta(\mathcal{I})$, while $\sigma_{t}$ and $\sigma_{\delta}$ can be computed from both $\bar{f}$ and $\mathcal{H}_{z}$. The rest of the paper will be devoted to applying this theory to a few specific cases, and to comparing the predictions with tracking simulations. For these we will assume that the longitudinal distribution is exponential in $\mathcal{H}_{z}$, with

$$
2 \pi \bar{f}(\mathcal{I})=A e^{-\mathcal{H}_{z}(\mathcal{I}) / \alpha_{c} \sigma_{\delta}^{2}}
$$

and $A$ fixed such that $\int d \mathcal{I} e^{-\mathcal{H}_{z}(\mathcal{I}) / \alpha_{c} \sigma_{\delta}^{2}}=1 / A$. We illustrate how the predictions may vary with different assumptions for $\bar{f}$ in Appendix D.

\section{HARMONIC POTENTIAL OF A SINGLE RF SYSTEM}

In this section we will assume that the longitudinal potential is given by the harmonic approximation of a single rf system, for which the Hamiltonian

$$
\mathcal{H}_{z}=\frac{\alpha_{c}}{2} p_{z}^{2}+\frac{\omega_{s}^{2}}{2 \alpha_{c} c^{2}} z^{2}
$$

where $\omega_{s}$ is the synchrotron frequency. The transformation to action-angle variables is well known (see, e.g., $[19,20]$ ) as

$$
z=\sqrt{\frac{2 \mathcal{I} \alpha_{c} c}{\omega_{s}}} \cos \Phi \quad p_{z}=-\sqrt{\frac{2 \mathcal{I} \omega_{s}}{\alpha_{c} c}} \sin \Phi,
$$

so that $\zeta(\mathcal{I})=\sqrt{2 \mathcal{I} \alpha_{c} c / \omega_{s}}$. Making the transformation (35) results in the unperturbed Hamiltonian $\mathcal{H}_{z}(\mathcal{I})=$ $\omega_{s} \mathcal{I} / c$, which in turn implies that the oscillation frequency is independent of action and that the unperturbed distribution function (33) is an exponential function of $\mathcal{I}$. In this case one can show that $\omega_{s}=\alpha_{c} \sigma_{\delta} / \sigma_{t}$ in equilibrium and that the quantities in (32) become

$$
\bar{f}(\mathcal{I})=\frac{e^{-\mathcal{I} / \sigma_{\delta} \sigma_{z}}}{2 \pi \sigma_{\delta} \sigma_{z}}, \quad \hat{\omega}=1, \quad \zeta(\mathcal{I})=\sigma_{z} \sqrt{\frac{2 \mathcal{I}}{\sigma_{\delta} \sigma_{z}}} .
$$

Then, we can integrate over action in (32) to get

$$
1=\frac{-i \hat{\lambda}}{1-e^{2 \pi i \hat{\Omega}}} \int_{0}^{2 \pi} d \theta e^{-k_{\xi}^{2} \sigma_{z}^{2}(1-\cos \theta)} e^{i \hat{\Omega} \theta} .
$$

This is closely related to the single-bunch result derived in [16], although it is significantly simpler since we only consider the first order Taylor series expansion of the longrange wakefield. Furthermore, in the Appendix we show that Eq. (37) is equivalent to the dispersion relation (C3), which was previously derived in Ref. [18]. Coupled-bunch stability can be found for a given long-range wakefield eigenvalue $\lambda$ and chromaticity $k_{\xi}=\xi \omega_{0} / \alpha_{c} c$ by solving (37) for $\Omega$; if $\Im(\Omega) \leq 0$ the system is stable, while an instability occurs if $\Im(\Omega)>0$. In the limit that the chromaticity vanishes we have $k_{\xi}=0$ and the integration can be easily done to find that

$$
\xi=0 \Rightarrow \Omega=\lambda
$$

Hence, at zero chromaticity the coupled-bunch growth rate equals the long-range eigenvalue $\lambda$. Note that this result can actually be derived from the general Eq. (28), so that in the zero chromaticity limit stability is independent of the longitudinal potential provided the long-range wakefield varies slowly over the bunch length. This is to be expected, since when $\xi=0$ the transverse motion is uncoupled from the synchrotron motion, and one cannot affect the other. On the other hand, at nonzero chromaticity the transverse betatron frequency depends upon the particle energy and therefore on its longitudinal position in the bunch, so that the collective oscillation is more complicated when $\xi \neq 0$.

We plot solutions to Eq. (32) with largest imaginary part as the diagonal crosses in Fig. 1, with panels (a) and (b) showing the instability growth rate $\Im(\hat{\Omega})$ as a function of $\Im(\hat{\lambda})$ for three values of the chromatic phase $k_{\xi} \sigma_{z}$; panel (a) assumes that $\Re(\lambda)=\Im(\lambda)$ as might be the case for the long-range resistive wall wake, while panel (b) uses $\Re(\hat{\lambda})=0$ as applies when the wakefield is due to a resonant higher-order rf-cavity mode. Although the former case with $\Re(\lambda)=\Im(\lambda)$ consistently has a somewhat larger instability growth rate, both panels show the same qualitative behavior. In particular, when $\xi \neq 0$ two specific regimes can be identified: the first applies when $\Im(\hat{\lambda}) \lesssim 3 k_{\xi} \sigma_{z} / 4$, and displays an instability growth rate $\Im(\hat{\Omega})$ that increases with $\Im(\hat{\lambda})$ at a rate inversely proportional to $k_{\xi} \sigma_{z}$; the 

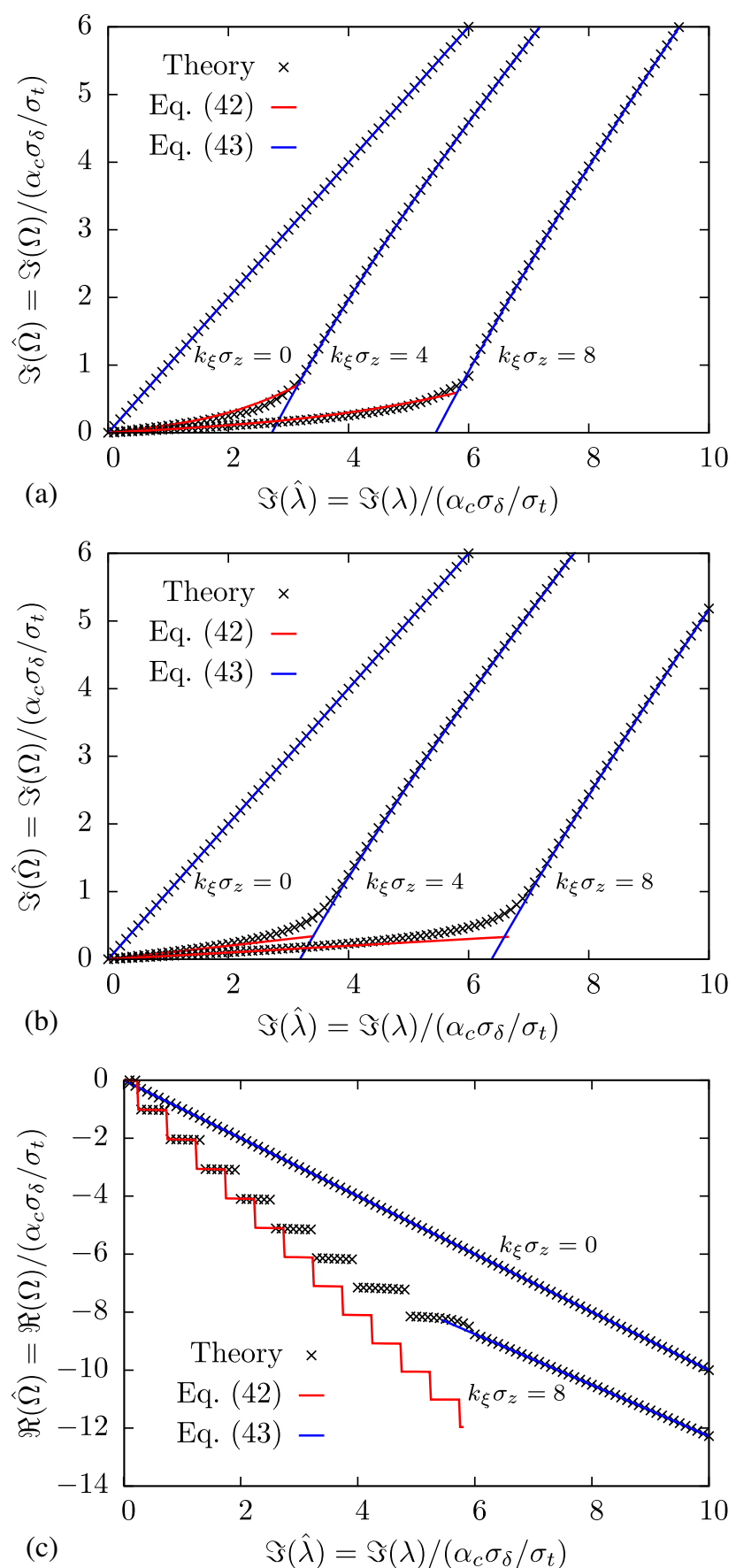

FIG. 1. Scaled complex instability frequency $\hat{\Omega}$ as a function of the scaled $\xi=0$ matrix growth rate $\Im(\hat{\lambda})$ for three values of the chromatic phase $k_{\xi} \sigma_{z}$. Panel (a) plots the instability growth rate assuming that $\Re(\lambda)=\Im(\lambda)$, where the diagonal crosses are the full theory Eq. (32), while the red and blue lines show the approximate, limiting Eqs. (42) and (43), respectively; panel (b) plots the same when the matrix eigenvalue is pure imaginary. Panel (c) plots the instability frequency $\Re(\hat{\Omega})$ when $\Re(\lambda)=\Im(\lambda)$, showing that the unstable mode oscillates at a harmonic of $\omega_{s}$ if $\lambda \lesssim 3 \xi \omega_{0} \sigma_{\delta} / 4$, but is not well described by a single synchrotron mode when Eq. (43) applies. second regime takes over when $\Im(\hat{\lambda}) \gtrsim 3 k_{\xi} \sigma_{z} / 4$, and predicts that the slope of the growth rate with $\Im(\hat{\lambda})$ is greater than (but comparable to) that for $\xi=0$.

We can understand these limiting behaviors in more detail using an approximate expression for Eq. (37), which we derive by splitting the integration region into one for $0 \leq \theta \leq \pi$ and the other for $\pi \leq \theta \leq 2 \pi$, changing variables in the second integral to $\theta^{\prime}=2 \pi-\theta$, and combining to write

$$
1=-i \hat{\lambda} \int_{0}^{\pi} d \theta e^{-k_{\xi}^{2} \sigma_{z}^{2}(1-\cos \theta)} \frac{e^{i \hat{\Omega} \theta}+e^{2 \pi i \hat{\Omega}} e^{-i \hat{\Omega} \theta}}{1-e^{2 \pi i \hat{\Omega}}}
$$

This is useful because in general we have either $k_{\xi} \sigma_{z} \gg 1$ or $\Im(\hat{\Omega}) \gtrsim 1$ or both, in which case the integrand is negligible for $\theta \gtrsim 1$ and the value of the integral is dominated by the region with $\theta \ll 1$. Then, we approximate $e^{-k_{\xi}^{2} \sigma_{z}^{2}(1-\cos \theta)} \approx e^{-k_{\xi}^{2} \sigma_{z}^{2} \theta^{2} / 2}$, extend the upper integration limit to $+\infty$, and integrate the result to obtain

$$
\begin{aligned}
1= & -\frac{i \hat{\lambda}}{k_{\xi} \sigma_{z}} \sqrt{\frac{\pi}{2}} \frac{e^{-\hat{\Omega}^{2} / 2 k_{\xi}^{2} \sigma_{z}^{2}}}{1-e^{2 \pi i \hat{\Omega}}} \\
& \times\left[\operatorname{erfc}\left(\frac{-i \hat{\Omega}}{\sqrt{2} k_{\xi} \sigma_{z}}\right)+e^{2 \pi i \hat{\Omega}} \operatorname{erfc}\left(\frac{i \hat{\Omega}}{\sqrt{2} k_{\xi} \sigma_{z}}\right)\right]
\end{aligned}
$$

alternatively, it may be more convenient to replace the complementary error function in favor of the Faddeeva function $w(z)=e^{-z^{2}} \operatorname{erfc}(-i z)$.

We have found that the solutions of the approximate (40) differ from Eq. (37) by less than $5 \%$ for any $k_{\xi} \sigma_{z}$ and $\hat{\lambda}$, but Eq. (40) has the advantage that it can be evaluated and solved using widely available mathematical packages. In addition, it provides a useful starting point to investigate the two limiting regimes identified in Fig. 1: the first where the chromatic effect significantly suppresses the transverse instability, and the second in which the chromaticity no longer provides a strong stabilizing force. We will often differentiate these two limits as "weak" or "strong," respectively, and in what follows consider each in turn.

\section{A. "Weak" instability limit}

The first limit of interest assumes that $\xi$ is large enough to significantly reduce the instability growth rate, so that both $k_{\xi} \sigma_{z} \gg 1$ and $\Im(\hat{\Omega}) \ll \Im(\hat{\lambda})$. We then expand (40) to second order in $\hat{\Omega} / k_{\xi} \sigma_{z}$ using $e^{-x^{2}}=1-x^{2} / 2+O\left(x^{4}\right)$ and $\operatorname{erfc}(x)=1-2 x / \sqrt{\pi}+O\left(x^{3}\right)$. After some algebra we find that

$$
\frac{\tan (\pi \hat{\Omega})}{\pi} \approx \frac{\hat{\lambda}}{\sqrt{2 \pi} k_{\xi} \sigma_{z}}\left(1+\frac{\hat{\Omega} \hat{\lambda}}{k_{\xi}^{2} \sigma_{z}^{2}}-\frac{\hat{\Omega}^{2}}{2 k_{\xi}^{2} \sigma_{z}^{2}}\right) .
$$


Note that the left-hand side is periodic and gives the same result for $\hat{\Omega}$ as for $\hat{\Omega}+m$ for integer $m$. Furthermore, we only expect the above relation to hold for small $\pi \hat{\Omega}$, so that Taylor expanding the left-hand side and setting $\hat{\Omega} \rightarrow m$ on the right-hand side implies that

$$
\hat{\Omega} \approx m+\frac{\hat{\lambda}}{\sqrt{2 \pi} k_{\xi} \sigma_{z}}\left[1+\frac{m}{k_{\xi}^{2} \sigma_{z}^{2}}\left(\hat{\lambda}-\frac{m}{2}\right)\right]
$$

for integer $m$.

The first thing Eq. (42) predicts is that the coupled-bunch growth rate can be reduced from its $\xi=0$ value by an amount proportional to the chromatic phase over the bunch. For small values of $\hat{\lambda}$ the reduction is by a factor $\sqrt{2 \pi} k_{\xi} \sigma_{z}$, which can be quite large. Additionally, the instability depends upon $m$ : for a sufficiently small coupled-bunch eigenvalue $\hat{\lambda}$ the mode with $m=0$ has the largest imaginary part, while a larger $\hat{\lambda}$ can lead to modes with higher $m$ being dominant, depending upon the nature of the coupledbunch instability (i.e., depending upon $\hat{\lambda}$ ). For example, if $\Re(\lambda)=0$ then the $m=0$ mode is always the most unstable, while if $\Re(\lambda)=\Im(\lambda)$ then Eq. (42) implies that the mode with $m$ equal to the integer part of $2\left[\Re(\hat{\lambda})-\frac{1}{4}\right]$ has the largest growth rate. Since $m$ identifies the integer part of $\Re(\hat{\Omega})$, these unstable modes oscillate with a higher frequency as they grow.

We summarize the predictions from Eq. (42) that were just described using the red solid lines in Figs. 1. In panels (a) and (b) these plot the largest instability growth rate $\Im(\hat{\Omega})$, while panel (c) plots the instability frequency $\Re(\hat{\Omega})$ for a long-range resistive wall wakefield that has $\Re(\lambda)=\Im(\lambda)$. The figures show that Eq. (42) applies provided $\Im(\hat{\lambda}) \lesssim 3 k_{\xi} \sigma_{z} / 4$ and $\Im(\hat{\Omega}) \ll 1$.

\section{B. "Strong" instability limit}

On the other hand, when the instability growth rate becomes of order the synchrotron frequency, $\Im(\hat{\Omega}) \gtrsim 1$, we have $\left|e^{2 \pi i \hat{\Omega} / \hat{\omega}(\mathcal{I})}\right| \ll 1$ and Eq. (40) simplifies to

$$
1=\frac{-i \hat{\lambda}}{k_{\xi} \sigma_{z}} \sqrt{\frac{\pi}{2}} e^{-\hat{\Omega}^{2} / 2 k_{\xi}^{2} \sigma_{z}^{2}} \operatorname{erfc}\left(\frac{-i \hat{\Omega}}{\sqrt{2} k_{\xi} \sigma_{z}}\right) .
$$

While solutions to Eq. (43) depend upon both the real and imaginary parts of $\lambda$, if $|\Re(\lambda)| \leq \Im(\lambda)$ as is typical, we find that Eq. (43) predicts that $\Im(\hat{\Omega})=0$ when the instability strength $\hat{\lambda} \approx(0.74 \pm 0.06) k_{\xi} \sigma_{z}$. Increasing $\Im(\hat{\lambda})$ beyond this range results in a similar (or larger) increase in the coupled-bunch instability growth rate, and the reduced slope given by (42) no longer applies. Hence, we find that the chromaticity is only effective in controlling the instability provided that

$$
\Im(\lambda) \lesssim \frac{3 k_{\xi} \sigma_{z}}{4} \frac{\alpha_{c} \sigma_{\delta}}{\sigma_{t}}=\frac{3}{4} \xi \omega_{0} \sigma_{\delta} .
$$

Taken together, our analysis has shown that the coupledbunch instability growth rate is reduced according to Eq. (42) if the spread in the betatron frequency due to chromatic effects $\xi \omega_{0} \sigma_{\delta}$ is much larger than the $\xi=0$ growth rate $\lambda$. This is the usual condition for phase mixing: the instability can be dramatically reduced when the spread in frequency is much greater than the instability growth rate. In contrast, phase mixing becomes less effective when $\Im(\lambda) \gtrsim \xi \omega_{0} \sigma_{\delta}$, in which case the growth rate is given by (44) and $\Im(\Omega)$ becomes significantly larger than that implied by (42).

We show the "strong instability" limit given by Eq. (43) using blue lines in Fig. 1. Panels (a) and (b) show how the growth rate increases quite rapidly once (44) no longer applies, while Fig. 1(c) shows that in this limit the frequency of the instability increases smoothly, and describing the unstable mode in terms of a single modal number is increasingly suspect.

\section{Connection to previous theories}

So far we have focused on the most unstable solution to the dispersion relation (37), since the solution with largest $\Im(\Omega)$ will ultimately determine multibunch stability. However, this approach somewhat obscures how to connect our theory to the more familiar mode-based theories of Sacherer and of Laclare. The former Sacherer theory [7] expands the distribution function in terms of a set of orthogonal azimuthal and radial modes, which in our case would result in a sum over Gauss-Laguerre functions. Hence, in terms of the scaled action $r=\mathcal{I} / \sigma_{z} \sigma_{\delta}$ we would expand the perturbation as

$$
\tilde{g}_{n}(r, \Phi) \propto \sum_{m, \ell} G_{n}^{m, \ell} e^{i m \Phi} e^{-r} r^{m / 2} L_{\ell}^{m}(r),
$$

which reduces the full theory to a matrix equation that in general has two mode indices [the azimuthal and radial numbers $(m, \ell)]$ to go along with the multibunch mode number $n$. The eigenvalues of the matrix equation yield a set of complex growth rates $\Omega$ that determine stability.

If we assume that the azimuthal and radial modes remain uncoupled in the presence of the wakefield then the eigenvalue problem reduces to the simple equation

$$
\hat{\Omega}_{p, m}=\omega_{s}+\hat{\lambda} \frac{e^{-k_{\xi}^{2} \sigma_{z}^{2}}\left(k_{\xi}^{2} \sigma_{z}^{2} / 2\right)^{2 p+m}}{p !(p+m) !} .
$$

This predicts a unique unstable growth rate for each value of $m$ and $p$. Unfortunately, solutions to the full matrix problem only display one unstable mode for each value of $m$ (the rest are stable or marginally stable), and also show that the unstable mode is typically a linear combination of 
many radial modes. The fact that the radial modes are strongly coupled is not surprising, since for a fixed synchrotron number $m$ all radial modes of the unperturbed problem are degenerate.

While the unperturbed problem does not distinguish between radial modes, it does separate the synchrotron modes by integral values of the synchrotron frequency. Hence, we expect the synchrotron modes to approximate the unstable system when the wakefield is sufficiently weak. In this case we should be able to apply Laclare's method [9] that solves for the $m$ th azimuthal component, and then assumes that the coupling between synchrotron modes can be neglected. In general this procedure results in an integral eigenvalue problem over the impedance, but by assuming that the long-range wakefield is constant over the bunch reduces Laclare's solution to

$$
\hat{\Omega}_{m}=m+\hat{\lambda} e^{-k_{\xi}^{2} \sigma_{z}^{2}} I_{m}\left(k_{\xi}^{2} \sigma_{z}^{2}\right) .
$$

This reduction of the growth rate was obtained in integral form by Ref. [26], and later as derived here by [18]. When $k_{\xi}^{2} \sigma_{z}^{2} \gg 1$ we can apply the asymptotic expansion $I_{m}(x) \sim e^{x} / \sqrt{2 \pi x}$, in which case we reproduce Eq. (42) provided $|\hat{\lambda}| \ll k_{\xi} \sigma_{z}$.

We illustrate how our theory reflects certain features of the azimuthal modes in Fig. 2, where we plot the complex growth rate of the nine modes with $0 \geq \mathfrak{R}(\hat{\Omega}) \geq-8.5$ as a function of the wakefield strength when $k_{\xi} \sigma_{z}=6$. Panel (a) plots the real part of $\hat{\Omega}$ as a function of the $\xi=0$ growth rate $\Im(\hat{\lambda})$, and shows that the scaled frequency slowly decreases from its integer value in the "weak" regime where $\Im(\lambda) \approx 3 k_{\xi} \sigma_{z} / 4$, beyond which the mode with $\Re(\hat{\Omega}) \approx-6$ shows a rapid decrease indicating that it is no longer welldescribed by a synchrotron mode.

Panels (b) and (c) of Fig. 2 plots the corresponding growth rates of the same modes. Panel (b) shows the significant suppression of the instability when $\Im(\lambda) \approx$ $3 k_{\xi} \sigma_{z} / 4$, followed by the significant increase of $\Im(\Omega)$ in the strong regime of the instability. Panel (c) plots the growth rate after subtracting the $\Im(\hat{\lambda}) / \sqrt{2 \pi} k_{\xi} \sigma_{z}$ characteristic of the weak regime. We see which mode is most unstable depends upon the wakefield strength, which is another way of describing what was previously plotted in Figs. 1(a) and (c).

\section{Comparison with tracking}

This section compares the theoretically predicted multibunch growth rates with those obtained from simulations using the particle tracking code ELEGANT [11]. Although the results will be presented in the same dimensionless form introduced in the previous section, the actual lattice parameters were chosen with an eye toward its eventual application to the APS-U storage ring, and are listed in
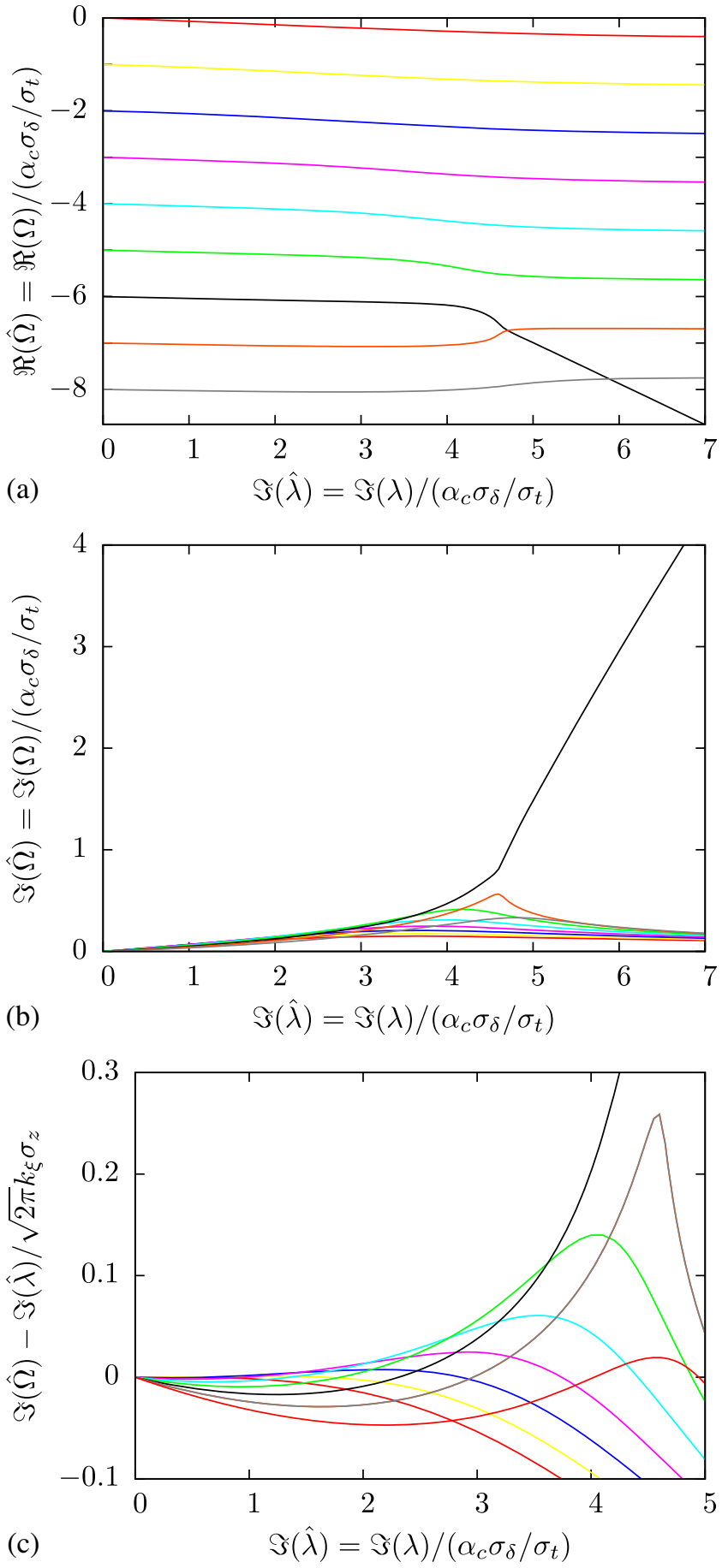

FIG. 2. Complex growth rate of the unstable modes when $k_{\xi} \sigma_{z}=6$. (a) plots the real part (frequency) of $\hat{\Omega}$ for nine different unstable modes, while (b) and (c) plot the corresponding growth rates.

Table I. There are two particular parameters from this list that we would like to highlight. The first is the matrix growth rate, whose strength we vary while maintaining the ratio $\Re(\lambda) / \Im(\lambda)$ that was derived for the long-range resistive wall wakefield assuming a fractional tune of 0.1 and a fill pattern of 48 equally-spaced bunches; since $\Omega$ 
TABLE I. Parameters used in ELEGANT simulations.

\begin{tabular}{lcc}
\hline \hline Parameter & Symbol & Value \\
\hline Vertical tune & $\nu_{y}$ & 36.1 \\
Chromaticity & $\xi_{y}$ & 0 to 5.5 \\
Momentum compaction & $\alpha_{c}$ & $4.04 \times 10^{-5}$ \\
Bunch length & $\sigma_{z}$ & $16.06 \mathrm{~mm}$ \\
Energy spread & $\sigma_{\delta}$ & $0.135 \%$ \\
Revolution time & $T_{0}$ & $3.68 \mathrm{~ms}$ \\
Synchrotron frequency & $\omega_{s} / 2 \pi$ & $160 \mathrm{~Hz}$ \\
Chromatic phase & $k_{\xi} \sigma_{z}$ & 0 to 12.5 \\
Number of bunches & $N_{b}$ & 48 \\
Total current & $I_{\text {tot }}$ & $200 \mathrm{~mA}$ \\
Vertical damping time & $\tau_{y}$ & $15.4 \mathrm{~ms}$ \\
Longitudinal damping time & $\tau_{z}$ & $20.5 \mathrm{~ms}$ \\
Coupled-bunch eigenvalue & $\lambda$ & $\Im(\lambda)(i-0.6)$ \\
\hline \hline
\end{tabular}

depends upon both the real and imaginary parts of $\lambda$ we provide further details of its calculation in Appendix E.

The second parameter of note from Table I is the bunch length $\sigma_{z}$ : the APS-U will employ a harmonic rf cavity to lengthen the bunch, which we simulate here using a single rf cavity whose voltage and harmonic number were chosen to match the rms bunch length $\sigma_{z}$ of the double rf system. We will revisit this approximation in Sec. IV, where we will see that the predictions for the growth rate given here for a harmonic oscillator agree well in the strong instability limit when $\Im(\Omega)>\alpha_{c} \sigma_{\delta} / \sigma_{t}$, namely, $\Im(\hat{\Omega})>1$, and otherwise only modestly overestimates the growth rate of a quartic potential with same rms bunch length. Further details of the ELEGANT simulations are in Appendix F.

In the first set of simulations we do not include the physics of damping and diffusion associated with the emission of sychrotron radiation. Hence, the simulated growth rates should be directly comparable to the Vlasov theory developed in Sec. II. We make this comparison for several different values of $k_{\xi} \sigma_{z}$ in Fig. 3(a), and find that the simulation and theory agree quite well over the entire range. In particular, the simulations clearly show the two regimes of multibunch stability that are distinguished by whether the $\xi=0$ growth rate $\Im(\lambda)$ is smaller or greater than the chromatic tune shift $\xi \omega_{0} \sigma_{\delta}$.

Figure 3(b) shows what happens when we add synchrotron radiation to the simulations, where for this comparison we have subtracted the vertical damping rate $1 /\left(\tau_{y} \omega_{s}\right) \approx$ 0.064 from the theory. The agreement is quite good when $k_{\xi} \sigma_{z} \lesssim 2$, but for higher chromaticities the simulations show no evidence of the weak instability limit described by Eq. (42), and are now stable when $\Im(\lambda) \lesssim 3 \xi \omega_{0} \sigma_{\delta} / 4$. On the other hand, in the strong instability limit that has $\Im(\lambda) \gtrsim 3 \xi \omega_{0} \sigma_{\delta} / 4$, the difference in simulated growth rates between panel (a) and (b) is approximately that of the vertical damping rate. Hence, at high chromaticity synchrotron emission can entirely eliminate the weak limit, in
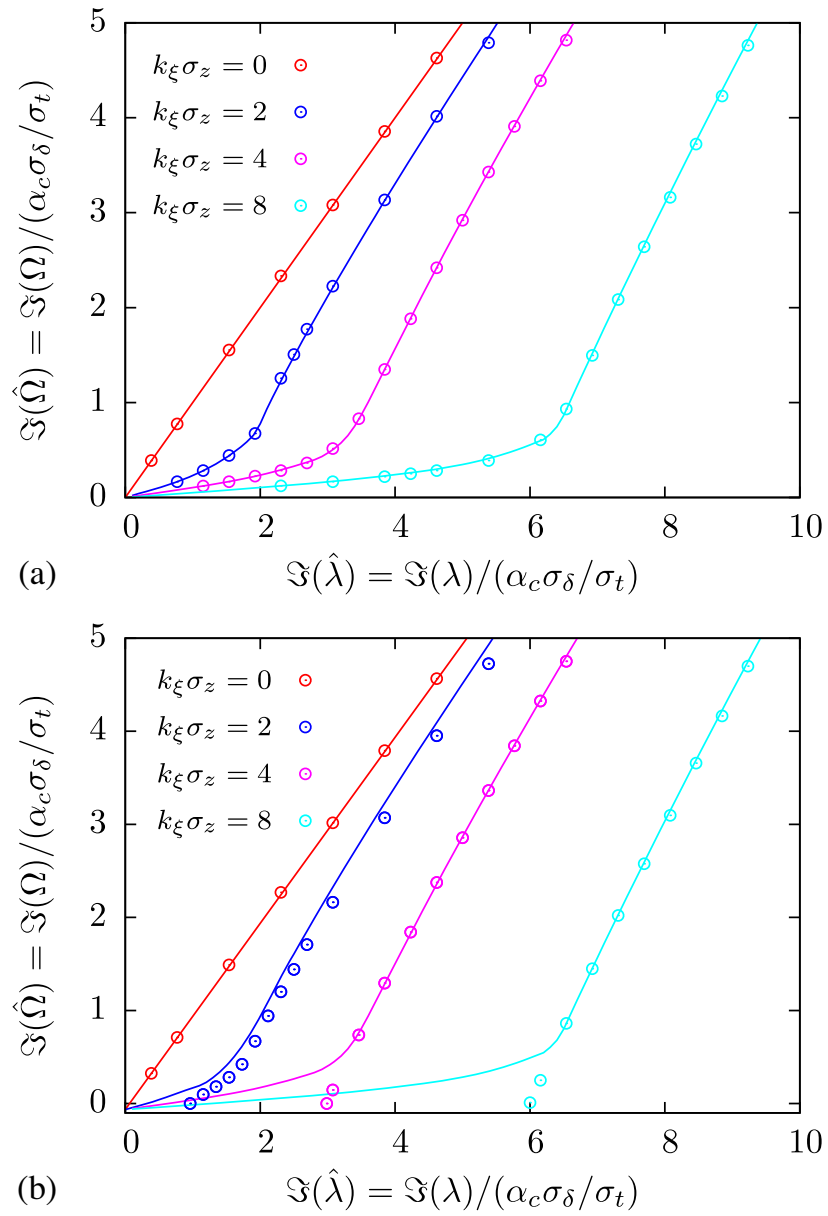

FIG. 3. Comparison of the growth rates obtained by tracking (dots) to those of theory (solid lines) when $V_{z} \propto z^{2}$. Tracking in panel (a) is Hamiltonian with no damping or diffusion, and we see very good agreement between theory and simulation. Panel (b) includes synchrotron emission in tracking as a stochastic process that leads to both damping and diffusion. In this case the additional physics of diffusion stabilizes the motion when the instability is weak and Eq. (42) applies; in contrast, when the growth rate is larger than the chromatic tune spread, $\Im(\lambda) \gtrsim 3 \xi \omega_{0} \sigma_{\delta} / 4$, tracking shows that diffusion does not affect the growth rate, and $\Im(\Omega)$ is approximately that given by Eq. (43) minus the scaled damping of 0.064 .

which case stability is well predicted by the strong limit Eq. (43) for all $\lambda$.

We will show that the disparity in the simulated growth rates observed between Figs. 3(a) and (b) is due to the stochastic nature of synchrotron emission, and that it is the diffusion in the longitudinal plane that stabilizes the motion at high chromaticity. To understand the physics prior to diving into the mathematics, we first note that the unstable transverse dipole mode can have significant longitudinal structure when $\xi \neq 0$. This is because the instability develops when the wakefield delivers kicks that are continually in phase with the particle's betatron oscillation, while the oscillation frequency depends both on the bare 
betatron tune and on the energy due to chromatic effects. Hence, the accumulated betatron phase will vary with longitudinal position as the particle makes synchrotron oscillations, and the instability will have a more complicated longitudinal structure as the chromaticity is increased.

Next, we recall the fact that diffusion due to synchrotron emission acts to smooth density perturbations along the energy $p_{z}$ with a characteristic smoothing time proportional to the square of the perturbation scale length. We therefore expect that diffusion will play an important stabilizing role on the higher-order synchrotron modes when $k_{\xi} \sigma_{z}>1$, and that this stabilization will be effective provided particles make many synchrotron oscillations during a growth time. In the next section we will derive an approximate dispersion relation that quantifies the effects of longitudinal damping and diffusion on stability. Since it is approximate, the dispersion relation in Sec. III E works best at moderate values of $k_{\xi} \sigma_{z}$; for the parameters in Fig. 3 the theory agrees with simulation to better than $1 \%$ when $k_{\xi} \sigma_{z} \lesssim 2$, and overestimates the onset of the strong regime by $\sim 5 \%$ when $k_{\xi} \sigma_{z}=4$, and by $\sim 20 \%$ when $k_{\xi} \sigma_{z}=8$.

\section{E. Instability theory including the longitudinal damping and diffusion}

We wish to add the physics of longitudinal damping and diffusion to our coupled-bunch stability theory. Unfortunately, the relatively simple-looking FokkerPlanck operator (18) becomes decidedly less so when we change variables from $\left(z, p_{z}\right)$ to the action-angle variables $(\Phi, \mathcal{I})$. To simplify matters somewhat we introduce the dimensionless action $r=\mathcal{I} / \sigma_{z} \sigma_{\delta}$, in terms of which we find that

$$
\begin{aligned}
\mathcal{E}_{\mathrm{FP}}^{\|}= & \frac{2}{c \tau_{z}}\left[r \frac{\partial^{2}}{\partial r^{2}}+(1+r) \frac{\partial}{\partial r}+\frac{1}{4 r} \frac{\partial^{2}}{\partial \Phi^{2}}+1\right] \\
& -\left\{\frac { e ^ { 2 i \Phi } } { c \tau _ { z } } \left[r \frac{\partial^{2}}{\partial r^{2}}+r \frac{\partial}{\partial r}-\frac{1}{4 r} \frac{\partial^{2}}{\partial \Phi^{2}}\right.\right. \\
& \left.\left.+i \frac{\partial^{2}}{\partial \Phi \partial r}+i \frac{r-1}{2 r} \frac{\partial}{\partial \Phi}\right]+ \text { c.c. }\right\} .
\end{aligned}
$$

Equation (22) with the right-hand-side given by (48) can be cast into an eigenvalue problem by expanding the perturbation as a sum of orthogonal modes as shown in Refs. [25,27]. Here, however, we choose to proceed with an approximate solution that is more in line with our Vlasov analysis, and that we believe gives another useful perspective to the stability problem.

Our approximate solution begins by expanding the perturbation in azimuthal (angular) modes as follows

$$
\tilde{g}_{n}(\Phi, \mathcal{I} ; \Omega)=\sum_{\ell} g_{n}^{\ell}(\mathcal{I} ; \Omega) e^{i \ell \Phi} .
$$

Inserting the expansion (49) into $\mathcal{E}_{\mathrm{FP}}^{\|}$results in the first line of (48) that is diagonal in the azimuthal index $\ell$, while the second two lines couple the mode $\ell$ to those at $\ell \pm 2$. We will neglect this latter coupling between modes in the Fokker-Planck operator, which applies provided the longitudinal damping is sufficiently weak for the given chromatic phase $k_{\xi} \sigma_{z}$. Next, we use the azimuthal expansion (49) in Eqs. (22) and (48), neglect the coupling between synchrotron modes in the Fokker-Planck operator $\mathcal{E}_{\mathrm{FP}}^{\|}$, and isolate the modes by multiplying by $e^{-i m \Phi} / 2 \pi$ and integrating over angle from 0 to $2 \pi$; for the simple harmonic potential we obtain

$$
\begin{gathered}
{\left[r \frac{d^{2}}{d r^{2}}+(1+r) \frac{d}{d r}-\frac{m^{2}}{4 r}+1-\frac{\tau_{z}}{2 i}\left(\Omega-m \omega_{s}\right)-\frac{\tau_{z}}{2 \tau_{y}}\right] g_{n}^{m}} \\
\quad=\frac{i \tau_{z} e^{-r}}{2} \int_{0}^{2 \pi} d \Phi \frac{e^{-i m \Phi-i k_{\xi} \sigma_{z} \sqrt{2 r} \cos \Phi}}{(2 \pi)^{2} \sigma_{z} \sigma_{\delta}} \sum_{j=0}^{N_{b}-1} \mathrm{M}_{n, j} \mathcal{D}_{j}(\Omega) \\
\quad=i \tau_{z} \frac{e^{-r} J_{m}\left(k_{\xi} \sigma_{z} \sqrt{2 r}\right)}{4 \pi i^{m} \sigma_{z} \sigma_{\delta}} \sum_{j=0}^{N_{b}-1} \mathrm{M}_{n, j} \mathcal{D}_{j}(\Omega)
\end{gathered}
$$

We would like to invert the differential operator on the left-hand side of (50). This can be done using a suitable Green function, and we show in Appendix B that the result of this inversion is

$$
\begin{aligned}
g_{n}^{m}= & \sum_{p=0}^{\infty} \frac{\left(k_{\xi} \sigma_{2} / \sqrt{2}\right)^{2 p+|m|} e^{k_{\xi}^{2} \sigma_{z}^{2} / 2}}{\Omega-m \omega_{s}+i / \tau_{y}+i(2 p+|m|) / \tau_{z}} \\
& \times \frac{e^{-r} r^{|m / 2|} L_{p}^{|m|}(r)}{2 \pi i^{m} \sigma_{z} \sigma_{\delta}(p+|m|) !} \sum_{j=0}^{N_{b}-1} \mathrm{M}_{n, j} \mathcal{D}_{j}(\Omega) .
\end{aligned}
$$

Now, we obtain an equation for the dipole moment $\mathcal{D}_{n}(\Omega)$ by multiplying by $e^{i m \Phi} e^{i k_{\xi} \sigma_{z} \sqrt{2 r} \cos \Phi}$, integrating over $(\Phi, \mathcal{I})=\left(\Phi, \sigma_{z} \sigma_{\delta} r\right)$, and summing over azimuthal index $m$. We take the phase space integrals using [28]

$$
\begin{gathered}
\int_{0}^{2 \pi} d \Phi e^{i m \Phi} e^{i x \cos \Phi}=2 \pi i^{m} J_{m}(x), \\
\int_{0}^{\infty} d r e^{-r} r^{m / 2} L_{p}^{m}(r) J_{m}(2 x \sqrt{r})=\frac{e^{-x^{2}}}{p !} x^{2 p+m},
\end{gathered}
$$

and diagonalize the wakefield matrix $\mathrm{M}$ as before to arrive at the dispersion relation

$$
\begin{aligned}
1= & \sum_{m=-\infty}^{\infty} \sum_{p=0}^{\infty} \frac{\lambda e^{-k_{\xi}^{2} \sigma_{z}^{2}}}{p !(p+|m|) !}\left(k_{\xi}^{2} \sigma_{z}^{2} / 2\right)^{2 p+|m|} \\
& \times\left[\Omega-m \omega_{s}+\frac{i}{\tau_{y}}+\frac{i}{\tau_{z}}(2 p+|m|)\right]^{-1} .
\end{aligned}
$$


The full dispersion relation (54) appears to have an additional dampinglike term $\sim(2 p+|m|) / \tau_{z}$ in the sum. Interestingly, the modal analysis of Ref. [25] identifies this same quantity as the additional damping associated with the synchrotron mode whose radial and azimuthal numbers are $(p, m)$. We argued in Ref. [27] that the physical origin of this mode number-dependent damping is the longitudinal diffusion, which tends to smooth out the rapidly varying higher-order modes more effectively than those of lower order. The advantage of the dispersion relation (54) is that all modes are taken together, with the only caveat that we neglected the modal coupling due to the Fokker-Planck operator itself.

To understand how Eq. (54) reflects the fact that higherorder modes are more readily damped, we first consider the Vlasov limit that obtains when the longitudinal damping is negligible. In this case we take $\tau_{z} \rightarrow \infty$ to find that the sum over $p$ equals the series representation for modified Bessel function $I_{|m|}\left(k_{\xi}^{2} \sigma_{z}^{2}\right)$, and we use the fact that $I_{|m|}(x)=I_{m}(x)$ for integer $m$ to derive the Vlasov limit

$$
1=\sum_{m=-\infty}^{\infty} \frac{\lambda e^{-k_{\xi}^{2} \sigma_{z}^{2}} I_{m}\left(k_{\xi}^{2} \sigma_{z}^{2}\right)}{\Omega-m \omega_{s}+i / \tau_{y}} .
$$

We show in the discussion leading up to (C3) that this is equivalent to our previously derived (37).

We have found that the dissipation-free result is recovered when the sum over $p$ in (54) converges sufficiently rapidly such that the terms with $(2 p+|m|) / \tau_{z} \gtrsim\left|\Omega-m \omega_{s}\right|$ contribute negligibly to the sum. This rapid convergence is typically guaranteed when the chromaticity is sufficiently small such that $k_{\xi} \sigma_{z} \lesssim 1$. Larger values of $k_{\xi} \sigma_{z}$ require ever more terms in the sum for it to converge, in which case the finite $\tau_{z}$ eventually plays a role.

We illustrate the effects of the longitudinal damping and diffusion on coupled bunch stability in Fig. 4, where we plot the ratio of the Fokker-Planck growth rate $\Im\left(\Omega_{\mathrm{FP}}\right)$ derived from (54) to that of the Vlasov limit Eq. (55) as a function of the $\xi=0$ dimensionless growth rate $\Im(\hat{\lambda})$. Figure 4 graphs this ratio for three values of chromatic phase $k_{\xi} \sigma_{z}$, and the solid lines have the scaled longitudinal damping $\tau_{z} \omega_{s}=1 / 500$ while the dashed lines use $\tau_{z} \omega_{s}=1 / 100$. We see that even relatively small longitudinal damping can significantly reduce the instability growth rate in the weak regime where $\Im(\hat{\lambda}) \lesssim 3 k_{\xi} \sigma_{z} / 4$, particularly as the chromatic phase becomes large. In addition, Fig. 4 predicts that the growth rate approaches its nominal, undamped value when $\Im(\hat{\lambda}) \gtrsim 3 k_{\xi} \sigma_{z} / 4$, which is consistent with the APS-U tracking results of Fig. 3(b).

To further explore the roles of diffusion and mode shape on stability, Fig. 5 shows three examples of the unstable perturbation in longitudinal phase space obtained from ELEGANT simulations of the long-range resistive wall instability. These phase portraits map how the unstable

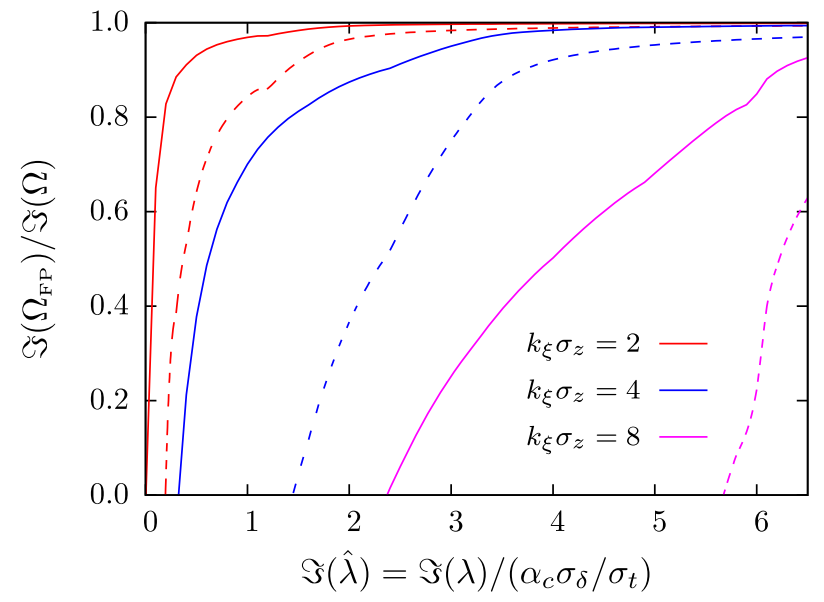

FIG. 4. Ratio of the theoretical growth rate including longitudinal damping to that with $\tau_{z} \rightarrow \infty$ as a function of the dimensionless growth rate at zero chromaticity $\Im(\hat{\lambda})$. Solid lines assume $\tau_{z} \omega_{s}=1 / 500$, while the dashed lines have $\tau_{z} \omega_{s}=1 / 100$.

betatron motion varies in the longitudinal plane, with red indicating particles that on average have a larger transverse displacement than the mean, while blue denotes a locally smaller betatron oscillation amplitude; we describe how they were obtained in Appendix F. Panels (a) and (b) in Fig. 5 compare the unstable mode profiles when the growth rate is smaller than $\omega_{s}$ to that when $\Im(\Omega)>\omega_{s}$ for the case with no synchrotron emission and $k_{\xi} \sigma_{z}=8$. When the instability is weak with a matrix growth rate $\Im(\hat{\lambda})=5.5<3 k_{\xi} \sigma_{z} / 4$, Fig. 5(a) shows that the unstable mode has the approximate azimuthal symmetry of a synchrotron mode. This is in stark contrast to the mode with nearly vertical stripes shown in Fig. 5(b), for which $\Im(\hat{\lambda})=7>3 k_{\xi} \sigma_{z} / 4$, the growth rate $\Im(\Omega)>\omega_{s}$, and Eq. (43) applies. Since diffusion acts to smooth gradients along $p_{z}$, its effect on the mode profile of Fig. 5(a) will be much stronger than that of Fig. 5(b). Indeed, simulations show that including the effects of synchrotron emission stabilizes the weak instability example in Fig. 5(a), even though the vertical damping rate is much smaller than the growth rate observed without synchrotron radiation, $1 / \tau_{y} \ll \Im(\Omega)$. On the other hand, when $\Im(\Omega)>\omega_{s}$ the unstable perturbation in Fig. 5(b) is a mixture of modes that limits the effect of diffusion and looks very similar to the one including synchrotron emission and shown in Fig. 5(c).

In summary, we have found that when the instability is strong such that $\Im(\lambda)>3 \xi \omega_{0} \sigma_{\delta} / 4$ and $\Im(\Omega) \gtrsim \omega_{s}$, stability is governed by Eq. (43) and the diffusion due to synchrotron emission plays essentially no role. When the instability is weak with $\Im(\lambda)<3 \xi \omega_{0} \sigma_{\delta} / 4$ and $\Im(\Omega) \ll \omega_{s}$, however, the unstable mode is approximately a synchrotron mode whose variations along $p_{z}$ tend to be smoothed by diffusion. In general these diffusive effects are proportional to the longitudinal damping rate and become more 

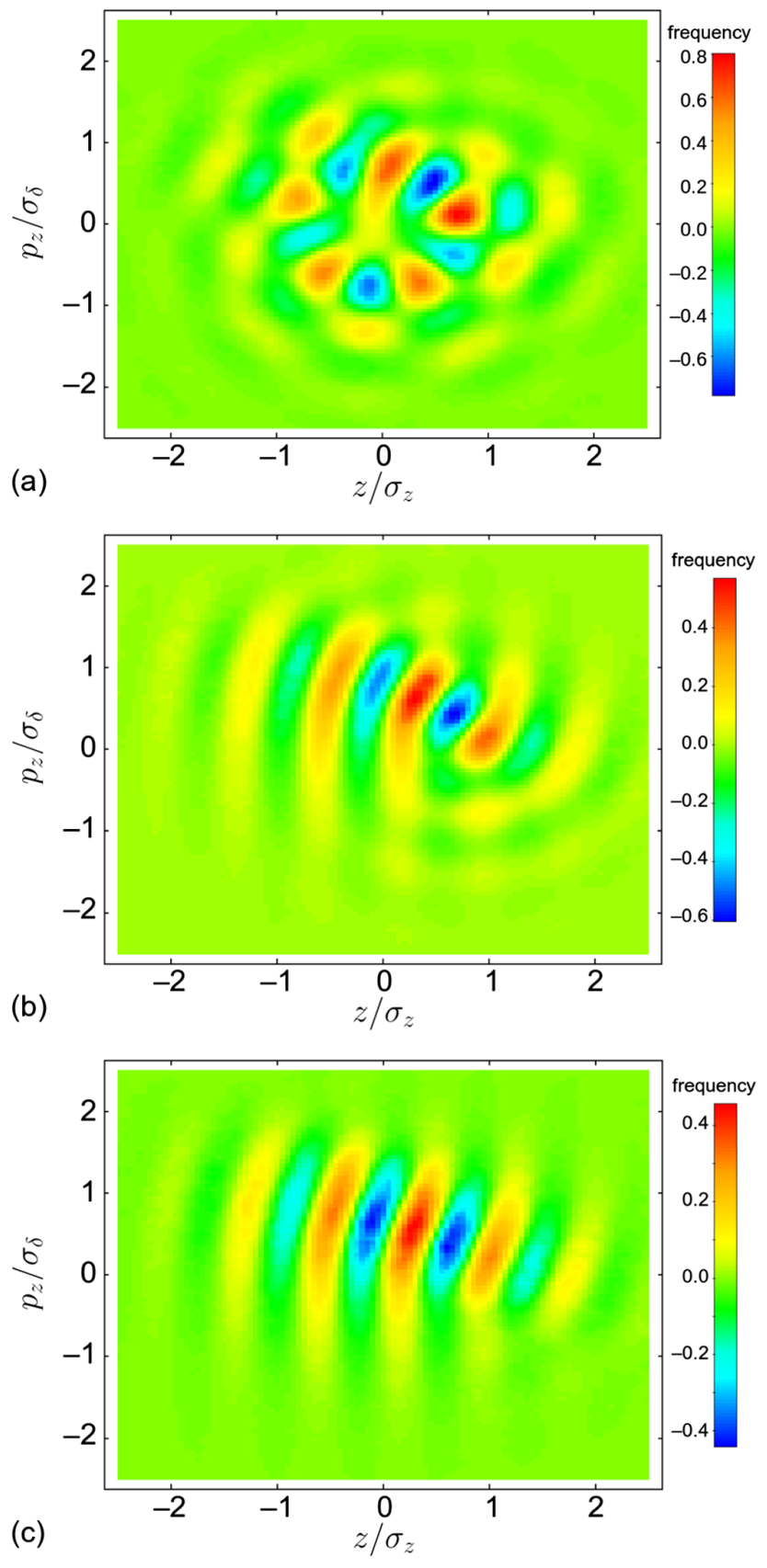

FIG. 5. Unstable longitudinal phase space density perturbations extracted from ELEGANT simulations for $k_{\xi} \sigma_{z}=8$. Panel (a) has no synchrotron emission and displays a complicated azimuthal dependence with $m \approx 5$ symmetry when $\Im(\hat{\lambda})=5$ and chromaticity plays an important role to control the instability. This contrasts with panel (b) for which the perturbation becomes more aligned with the $p_{z}$ axis as the wakefield strength increases such that $\Im(\hat{\lambda})=7>3 k_{\xi} \sigma_{z} / 4$. Panel (c) has the same parameters as (b) but also includes synchrotron emission.

significant at large $k_{\xi} \sigma_{z}$; more precise numerical predictions can be obtained with the dispersion relation (54). For the parameters of the APS-U listed in Table I, the damping and diffusion of synchrotron emission completely eliminates the "weak" instability provided $k_{\xi} \sigma_{z} \gtrsim 3$.

\section{QUARTIC POTENTIAL OF AN IDEALLY TUNED DOUBLE RF SYSTEM}

Many of the next generation ultralow emittance storage rings, including MAX-IV [29,30], APS-U [31], and ALS-U [32], rely (or plan to rely) on a higher harmonic rf system to stretch the bunch and reduce the particle density, thereby increasing the lifetime and decreasing emittance growth due to intrabeam scattering. What is often called "optimal stretching" is achieved when the two rf systems are tuned such that the longitudinal potential near the equilibrium point becomes a quartic function of position, $V_{z}(z) \propto z^{4}$. To be more explicit, if one starts with a main cavity of fundamental frequency $\omega_{\mathrm{rf}}$, and then adds to that a harmonic cavity with frequency $h \omega_{\mathrm{rf}}$ for integer $h$, then the total potential is

$V_{z}=V_{1} \sin \left(\omega_{\mathrm{rf}} z / c+\phi_{1}\right)+V_{h} \sin \left(h \omega_{\mathrm{rf}} z / c+\phi_{h}\right)$,

where $V_{1, h}$ and $\phi_{1, h}$ are the main and harmonic voltages and phases, respectively.

We now Taylor expand Eq. (56) for small displacements about the equilibrium where $\left|h \omega_{\mathrm{rf}} z / c\right| \ll 1$, and find that for a given main voltage $V_{1}$ we can choose the parameters $V_{h} / V_{1}, \phi_{1}$, and $\phi_{h}$ such that the linear term balances the average energy loss per turn while the quadratic and cubic terms vanish, thereby obtaining a quartic potential. Having done this, we again assume that the equilibrium $\bar{f}(\mathcal{I}) \propto e^{-\mathcal{H}_{z} / \alpha_{c} \sigma_{\delta}^{2}}$, so that we can write the longitudinal $\mathcal{H}_{z}$ in terms of the rms bunch length $\sigma_{z}$ and energy spread $\sigma_{\delta}$ as

$$
\begin{aligned}
\mathcal{H}_{z}\left(z, p_{z}\right) & =\frac{\alpha_{c}}{2} p_{z}^{2}+\frac{\alpha_{c} \sigma_{\delta}^{2} \Gamma(3 / 4)^{2}}{\sigma_{z}^{4} \Gamma(1 / 4)^{2}} z^{4} \\
& =\alpha_{c} \sigma_{\delta}^{2}\left[\frac{\sqrt{\pi} \Gamma(3 / 4)^{3 / 2}}{\sqrt{2} \Gamma(1 / 4)^{3 / 2}} \frac{3 \mathcal{I}}{\sigma_{\delta} \sigma_{z}}\right]^{4 / 3},
\end{aligned}
$$

where $\Gamma(x)$ is the usual Gamma function and the second line gives the equilibrium Hamiltonian as a function of the action $\mathcal{I}$; details can be found in Ref. [33].

Now, we investigate to what extent the nonlinear Hamiltonian (58) affects multibunch transverse stability. We have already shown that transverse stability is independent of the longitudinal dynamics at zero chromaticity, and the focus here will be on $\xi \neq 0$. We will find that the phenomenology of coupled-bunch stability is quite similar to that derived in the previous section, but with somewhat more complicated details. In particular, we will again be able to identify "weak" and "strong" limits of the instability that are distinguished by whether the $\xi=0$ growth rate $\Im(\lambda)$ is smaller or larger than the chromatic tune spread $\xi \omega_{0} \sigma_{\delta}$.

Prior to this theoretical investigation, we will introduce a different but equivalent expression to Eq. (32) that we found more convenient for numerical work in the nonlinear 
quartic potential. First, recall that we will require expressions for $\bar{f}, \zeta$, and $\hat{\omega}$; using the Hamiltonian (58) we find that the longitudinal equilibrium is

$$
\begin{gathered}
2 \pi \bar{f}(\mathcal{I})=\frac{4 a}{3 \Gamma(3 / 4)} \exp \left[-(a \mathcal{I})^{4 / 3}\right], \\
\text { with } \quad a=\frac{3 \sqrt{\pi} \Gamma(3 / 4)^{3 / 2}}{\sqrt{2} \Gamma(1 / 4)^{3 / 2} \sigma_{\delta} \sigma_{z}} \approx \frac{0.7388}{\sigma_{\delta} \sigma_{z}},
\end{gathered}
$$

while the quartic oscillator has a scaled oscillation frequency $\hat{\omega}$ and Fourier coefficient $\zeta$ given by [33]

$$
\begin{gathered}
\hat{\omega}(\mathcal{I})=\frac{2 \sqrt{2 \pi} \Gamma(3 / 4)^{3 / 2}}{\Gamma(1 / 4)^{3 / 2}}(a \mathcal{I})^{1 / 3}, \\
\zeta(\mathcal{I})=\sigma_{z} \frac{4 \sqrt{\pi \Gamma(3 / 4)}}{\cosh (\pi / 2) \sqrt{\Gamma(1 / 4)}}(a \mathcal{I})^{1 / 3} .
\end{gathered}
$$

Then, we show in Appendix $C$ that multibunch stability is governed by Eq. (C2), which we rewrite here as

$$
1=\hat{\lambda} \int_{0}^{\infty} d \mathcal{I} 2 \pi \bar{f}(\mathcal{I}) \sum_{m=-\infty}^{\infty} \frac{J_{m}\left[k_{\xi} \zeta(\mathcal{I})\right]^{2}}{\hat{\Omega}-m \hat{\omega}(\mathcal{I})} .
$$

The dispersion relation (63) is faster to solve numerically than (32) because it has exchanged one integration for an infinite sum, and we have found that the sum achieves sufficient convergence for all parameters considered here if we truncate it to $|m| \leq 25$. Equation (63) applies provided $\Im(\Omega)>0$; when $\Im(\Omega) \leq 0$ we in general have to deform the integration contour as prescribed by Landau to account for the poles in the integrand [34]. However, for small values of the wakefield strength we find that $|\hat{\Omega}| \ll 1$, in which case the dispersion relation is dominated by the $m=0$ term of the sum in Eq. (63), while the contributions from the poles when $m \neq 0$ become vanishingly small. Hence, when $|\hat{\lambda}| \ll 1$ we have

$$
\hat{\Omega} \approx \hat{\lambda} \int_{0}^{\infty} d \mathcal{I} 2 \pi \bar{f}(\mathcal{I}) J_{0}\left[k_{\xi} \zeta(\mathcal{I})\right]^{2},
$$

and Landau damping plays essentially no additional role.

We show several representative solutions to (63) for the coupled-bunch growth rates $\Im(\Omega)$ in a quartic longitudinal potential in Fig. 6. These results assume the basic parameters listed in Table I, in particular using an identical lattice, bunch pattern, and long-range resistive wall wakefield so that $\lambda=\Im(\lambda)(i-0.6)$, and furthermore assumes the same single bunch $\sigma_{z}$ and $\sigma_{\delta}$ (the one distinction from the Table is that the quartic potential does not have a unique synchrotron frequency $\omega_{s}$ ). The graph in Fig. 6(a) displays the same features as does the simple harmonic potential in the previous section, namely, a significant reduction in the dependence of $\Im(\Omega)$ with $\Im(\lambda)$ when the $\xi=0$ growth rate is much smaller than the chromatic tune
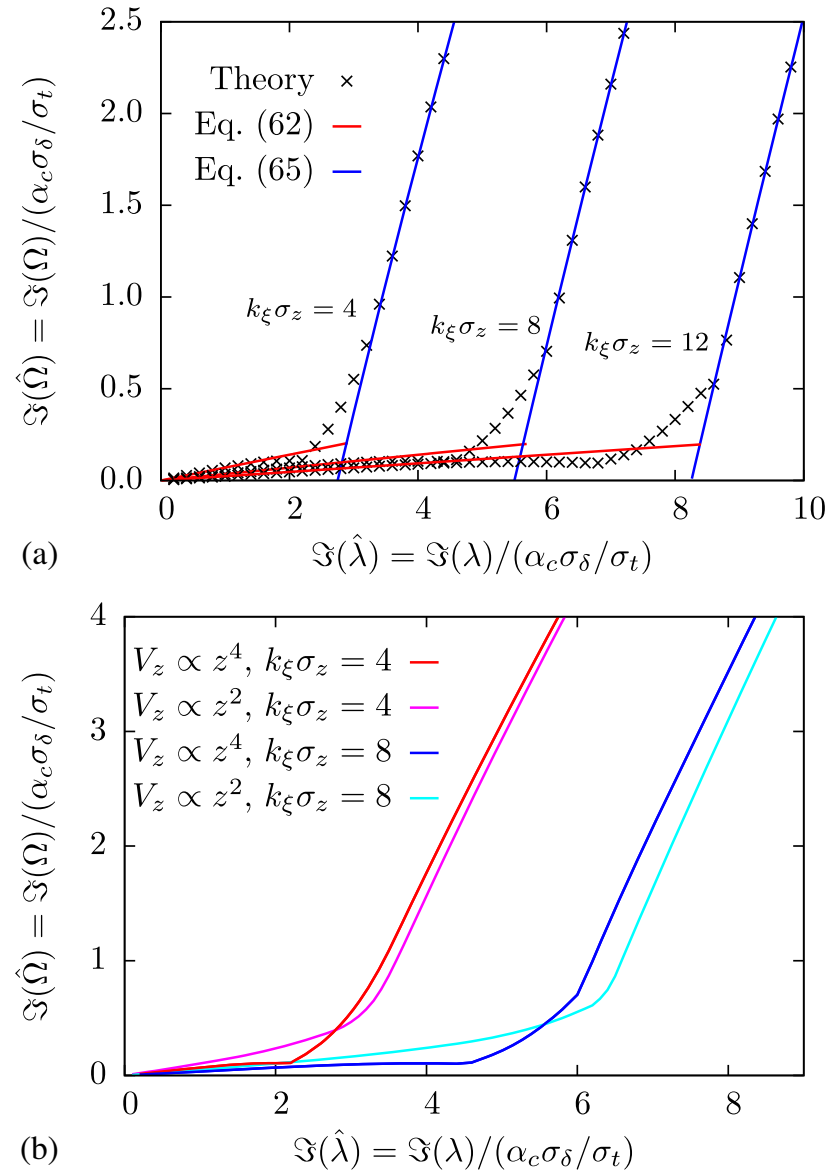

FIG. 6. (a) Theoretically predicted growth rates for the quartic potential as a function of the $\xi=0$ growth rate $\Im(\lambda)$. Crosses are the full theory, while the red and blue lines represent the weak and strong instability limits, respectively. (b) Comparison of the growth rates between the quartic (red and blue) and the quadratic (magenta and cyan) longitudinal potentials, for two different values of the chromaticity.

spread, followed by a dramatic increase in the slope when $\Im(\lambda) \gtrsim \xi \omega_{0} \sigma_{\delta}$.

Figure 6(b) directly compares the maximum long-range resistive wall growth rates in a quartic and harmonic potential at two different values of $k_{\xi} \sigma_{\delta}$. We see that when $\Im(\lambda) \ll \xi \omega_{0} \sigma_{\delta}$ the quartic potential reduces $\Im(\lambda)$ below the value predicted in the quadratic potential, while in the strong instability limit the growth rates are very similar. To make these statements more quantitative, we turn to analyzing the dispersion relation Eq. (39) in the weak and strong limits.

\section{A. Weak and strong instability regimes}

We begin with the weak limit which obtains when both $|\hat{\Omega}| \ll 1$ and $k_{\xi} \sigma_{z} \gg 1$. Then, we expand the exponentials in (32) assuming that $|\Omega / \omega(\mathcal{I})| \ll 1$, in which case the subsequent integration over $\theta$ is symmetric about $\theta=\pi$ and we obtain 


$$
\begin{aligned}
1 & \approx \frac{\hat{\lambda}}{\hat{\Omega}} \int_{0}^{\infty} d \mathcal{I} \bar{f}(\mathcal{I}) \int_{0}^{\pi} d \theta 2 J_{0}\left[2 k_{\xi} \zeta(\mathcal{I}) \sin (\theta / 2)\right] \\
& \approx \frac{2 \hat{\lambda}}{\hat{\Omega}} \int_{0}^{\infty} d \overline{\mathcal{I}}(\mathcal{I}) \int_{0}^{\pi} d \theta J_{0}\left[k_{\xi} \zeta(\mathcal{I}) \theta\right] \\
& =\frac{\hat{\lambda}}{\hat{\Omega}} \int_{0}^{\infty} d \mathcal{I} 2 \pi \bar{f}(\mathcal{I})_{1} F_{2}\left[\frac{1}{2} ; 1, \frac{3}{2} ;-k_{\xi}^{2} \zeta(\mathcal{I})^{2} \pi^{2} / 4\right] \\
& \approx \frac{\hat{\lambda}}{\hat{\Omega}} \int_{0}^{\infty} d \mathcal{I} 2 \pi \frac{\bar{f}(\mathcal{I})}{\pi k_{\xi} \zeta(\mathcal{I})} .
\end{aligned}
$$

The second line uses $\sin (\theta / 2) \approx \theta / 2$ which can be applied provided $k_{\xi} \sigma_{z} \gg 1$, while the third line involves a hypergeometic function [35] that we expand for large argument using ${ }_{1} F_{2}\left(1 / 2 ; 1,3 / 2 ;-x^{2} / 4\right)=x^{-1 / 2}+O\left(x^{-3 / 2}\right)$. Inserting the quartic equilibrium distribution (59) and Fourier coefficient (62) into (65) results in an integral we can evaluate. Hence,

$$
\hat{\Omega}=\frac{\cosh (\pi / 2) \sqrt{\Gamma(1 / 4)}}{4 \pi \Gamma(3 / 4)^{3 / 2}} \frac{\hat{\lambda}}{k_{\xi} \sigma_{z}} \approx 0.703 \frac{\hat{\lambda}}{\sqrt{2 \pi} k_{\xi} \sigma_{z}}
$$

if the instability is weak and $|\hat{\Omega}| \ll 1$. We plot Eq. (66) as red lines in Fig. 6(a), which again shows that the weak limit can be used if $\Im(\lambda) \lesssim 3 \xi \omega_{0} \sigma_{\delta} / 4$.

Equation (66) predicts that chromatic effects reduce the growth rate by an amount inversely proportional to the head-tail phase $k_{\xi} \sigma_{z}$ when $|\Omega| \ll \alpha_{c} \sigma_{\delta} / \sigma_{t}$ (or equivalently when $\left.|\lambda| \ll \xi \omega_{0} \sigma_{\delta}\right)$. Hence, both the quartic and quadratic potentials exhibit a similar weak instability regime when the $\xi=0$ growth rate is much less than the chromatic tune shift over the bunch. If we directly compare Eqs. (66) and (42) we find that the growth rate of the flattened quartic potential is about $70 \%$ that of the harmonic potential when $\Im(\Omega) \ll 1$. Additionally, Fig. 6(b) shows that the difference between the two growth rates increases as a function of the coupledbunch instability when the weak limit still applies. These predictions are broadly consistent with the theoretical and tracking results of Ref. [10].

Now, we turn to deriving an expression for the complex coupled-bunch frequency $\Omega$ when the instability is strong. This case is defined by $\Im(\hat{\Omega}) \gg 1$, in which case we can neglect the small quantity $e^{2 \pi i \hat{\Omega}}$ in the solution Eq. (59). Furthermore, we have $\left|e^{i \hat{\Omega} \theta / \hat{\omega}}\right| \ll 1$ unless $|\theta| \ll 1$, so that we expand $\sin (\theta / 2) \approx \theta / 2$ and extend the integration region over $\theta$ to infinity to obtain

$$
\begin{aligned}
1 & \approx-i \hat{\lambda} \int_{0}^{\infty} d \mathcal{I} \frac{2 \pi \bar{f}(\mathcal{I})}{\hat{\omega}(\mathcal{I})} \int_{0}^{\infty} d \theta J_{0}\left[k_{\xi} \zeta(\mathcal{I}) \theta\right] e^{i \hat{\Omega} \theta / \hat{\omega}(\mathcal{I})} \\
& =-\frac{i \hat{\lambda}}{k_{\xi} \sigma_{z}} \int_{0}^{\infty} d \mathcal{I} \frac{2 \pi \bar{f}(\mathcal{I})}{\sqrt{[\hat{\omega}(\mathcal{I}) \zeta(\mathcal{I})]^{2}-\left(\hat{\Omega} / k_{\xi} \sigma_{z}\right)^{2}}}
\end{aligned}
$$

The last part of the calculation involves inserting the equilibrium distribution $\bar{f}$, scaled oscillation frequency $\hat{\omega}$, and Fourier coefficient $\zeta$ of the quartic oscillator into (67). We use Eqs. (59)-(62), and define the dimensionless integration variable $x=a \mathcal{I}$ and the quantity

$$
b=\frac{\cosh (\pi / 2) \Gamma(1 / 4)^{2}}{8 \pi \sqrt{2} \Gamma(3 / 4)^{2}} \approx 0.618
$$

Then, the integration in (67) becomes

$$
\begin{aligned}
1 & =-b \frac{i \hat{\lambda}}{k_{\xi} \sigma_{z}} \int_{0}^{\infty} d x \frac{\frac{4}{3 \Gamma(3 / 4)} e^{-x^{4 / 3}}}{\sqrt{x^{4 / 3}-\left(b \hat{\Omega}^{2} / k_{\xi} \sigma_{z}\right)^{2}}} \\
& =-b \frac{i \hat{\lambda}}{k_{\xi} \sigma_{z}} U\left(\frac{1}{2}, \frac{3}{4},-\frac{b^{2} \hat{\Omega}^{2}}{k_{\xi}^{2} \sigma_{z}^{2}}\right),
\end{aligned}
$$

where $U$ is the confluent hypergeometric function of the second kind [28] (also called the Tricomi function).

Solutions to (69) are given by the blue lines in Fig. 6(a), which we see agree quite well with the full theory when $\Im(\hat{\lambda}) \gtrsim 3 k_{\xi} \sigma_{z} / 4$. In this strong limit we have found that the coupled-bunch motion appears to be largely independent of the longitudinal potential; for a given $k_{\xi} \sigma_{z}$ the complex frequency $\Omega$ is nearly the same if $V_{z} \propto z^{4}$ as it is when $V_{z} \propto z^{2}$.

\section{B. Comparison of theory to tracking}

Now we turn to comparing the theory just presented to results derived from the tracking code ELEGANT. As done in Sec. IV B, the basic lattice parameters were chosen to model the APS Upgrade multibend achromat design, and are listed in Table I. In this case, however, the tracking includes the planned double rf system, which is modeled as two prescribed accelerating voltages and phases applied once per turn; the rf parameters are listed in Table II.

As in the previous section, we will first compare the predictions of our Vlasov theory with Hamiltonian simulations that neglect the diffusion due to synchrotron emission. There are (at least) two ways we can do this: (1) eliminate synchrotron emission entirely or (2) model it as a single energy loss given to all particles once per turn.

TABLE II. Parameters of the double rf system used in ELEGANT simulations.

\begin{tabular}{lcc}
\hline \hline Parameter & Symbol & Value \\
\hline Main rf voltage & $V_{\mathrm{rf}}$ & $4.6 \mathrm{MV}$ \\
Main rf frequency & $f_{\mathrm{rf}}$ & $352.06 \mathrm{MHz}$ \\
Main rf phase & $\phi_{1}$ & $138.3^{\circ}$ \\
Harmonic number & $h$ & 4 \\
Harmonic voltage & $V_{4}$ & $0.88 \mathrm{MV}$ \\
Harmonic phase & $\phi_{4}$ & $347.4^{\circ}$ \\
\hline \hline
\end{tabular}


The former method requires changing the rf parameters to maintain the flattened (quartic) potential, while the latter eliminates diffusion but retains the transverse damping. We have decided to adopt the second method since it easily enables us to have identical longitudinal rf potentials for all tracking simulations, which also means that in subsequent comparisons we will also subtract the transverse damping rate $1 / \tau_{y}$ from the theory.

We ran a number of ELEGANT simulations using the lattice parameters from Table I and the $V_{z} \propto z^{4}$ rf system from Table II, to which we added a long-range resistive wall wakefield of various strengths. The multibunch growth rate was extracted from the simulation by fitting an exponential to both the centroid motion $\langle y\rangle$ and the rms width $\sigma_{y}$. The growth rates from tracking are compared to theoretical predictions in Fig. 7(a), where the theory includes the scaled transverse damping by subtracting off $\tau_{y}^{-1} /\left(\alpha_{c} \sigma_{\delta} / \sigma_{t}\right) \approx 0.064$. Again, the behavior is quite similar to that when the longitudinal potential is simple harmonic: the growth rates are reduced by an amount $\sim 0.28 / k_{\xi} \sigma_{z}$ when the $\xi=0$ growth rate is less than the chromatic tune spread over the bunch, $\Im(\lambda) \gtrsim 3 \xi \omega_{0} \sigma_{\delta} / 4$; for larger values of $\Im(\lambda)$ the slope of $\Im(\hat{\Omega})$ vs $\Im(\hat{\lambda})$ is of the order of one.

In addition, Fig. 7(b) presents a comparison at $k_{\xi} \sigma_{z}=8$ between the quadratic and quartic potential predictions for the unstable mode's oscillation frequency shift from $\omega_{\beta}$, namely, $\Re(\Omega)$. When the instability is weak with $\Im(\lambda) \lesssim 3 \xi \omega_{0} \sigma_{\delta} / 4$, Fig. 7(b) shows that frequency difference is nearly an integer multiple of $\alpha_{c} \sigma_{\delta} / \sigma_{t}$, which is consistent with the unstable mode being well-approximated by a synchrotron mode. In the quadratic potential both the theory (red line) and the simulation (magenta points) indicate that $\Re(\hat{\Omega})$ decreases with $\Im(\hat{\lambda})$ in a steplike fashion with an average slope $\approx-1$. On the other hand, when $V_{z} \propto z^{4}$ both theory (blue line) and simulation (cyan points) predict that the frequency shift is approximately zero. This was anticipated by Ref. [10], and is physically due to the fact that the synchrotron frequency in a quartic potential varies with amplitude, so that synchrotron oscillations tend to mix modes that have nontrivial angular dependence.

When the instability is strong, $\Im(\lambda) \gtrsim 3 \xi \omega_{0} \sigma_{\delta} / 4$ and the growth rate is larger than the typical synchrotron oscillation frequency, Fig. 7(b) shows that $\Re(\Omega)$ is no longer an integer multiple of $\alpha_{c} \sigma_{\delta} / \sigma_{t}$; rather, in this limit the frequency difference decreases smoothly as a function of $\Im(\lambda)$, with $\Re(\hat{\Omega}) \sim \Im(\hat{\lambda})$. Furthermore, Fig. 7(b) shows that the frequency shift of the strongly unstable mode is approximately the same for the quadratic and quartic potentials, which is further evidence that in this limit the details of the longitudinal potential no longer impact transverse multibunch stability.

The multibunch instability dynamics can be further illuminated by inspecting the longitudinal phase space of the unstable mode. We plot these for the quartic potential
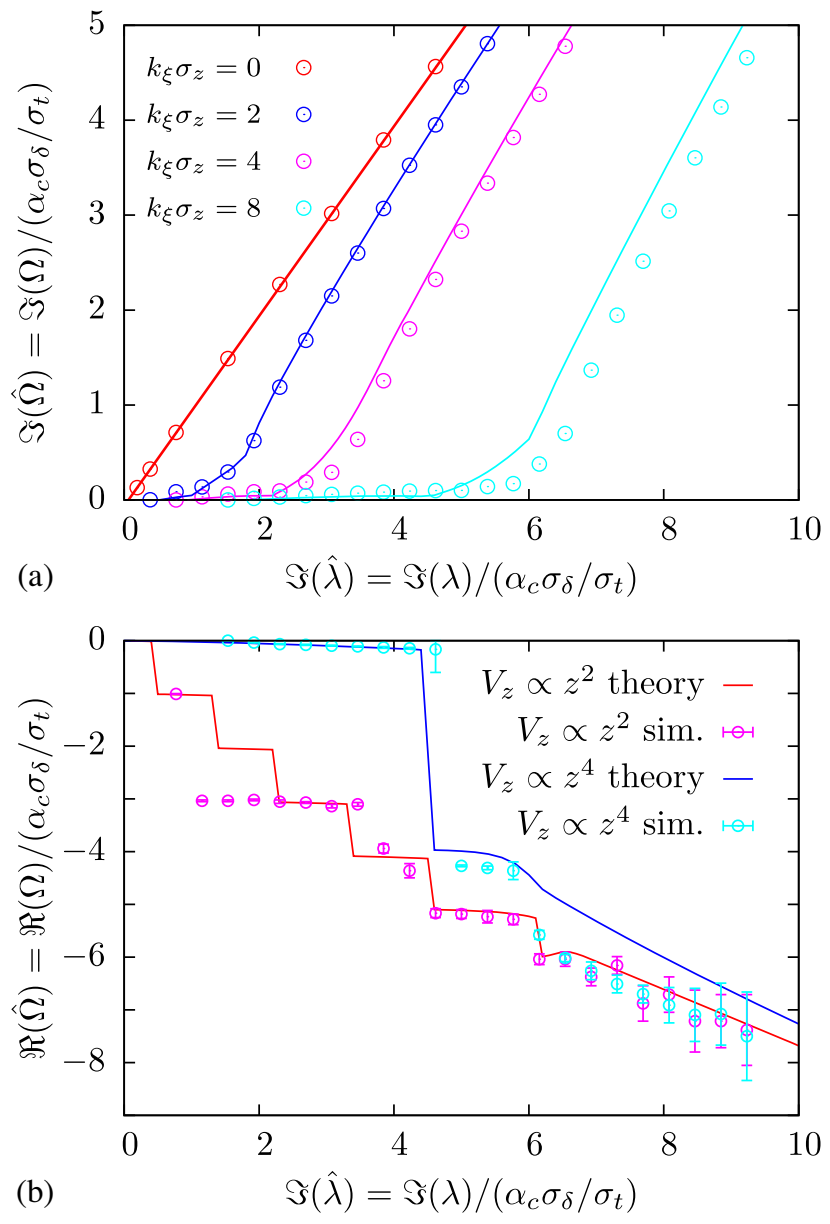

FIG. 7. Comparison of the unstable dynamics obtained by tracking (dots) to those of theory (solid lines) when the longitudinal damping and diffusion are neglected. Panel (a) compares the predicted multibunch growth rate in the quartic potential $V_{z} \propto z^{4}$ for four different values of $k_{\xi} \sigma_{z}$. The comparison is rather good and quite similar to that in Fig. 3(a). Panel (b) plots the unstable frequency shift from $\omega_{\beta}$ when $k_{\xi} \sigma_{z}=8$ for the quadratic potential (red and magenta) and the quartic potential (blue, cyan). The frequency shift is approximately an integer multiple of $\alpha_{c} \sigma_{\delta} / \sigma_{t}$ when $\Im(\lambda) \lesssim 3 \xi \omega_{0} \sigma_{\delta} / 4$, while for larger values of the instability strength $\mathfrak{R}(\Omega)$ smoothly decreases with $\Im(\lambda)$ and is largely independent of $V_{z}$.

and chromatic phase $k_{\xi} \sigma_{z}=8$ in Fig. 8; note that the only difference between these results and those of Fig. 5 is the shape of the longitudinal potential. In particular, panels (a), (b), and (c) have the same resistive wall strength and $k_{\xi} \sigma_{z}$ as do the corresponding plots in Fig. 5, and panels (a) and (b) of Fig. 8 here neglect synchrotron emission while (c) includes it.

Figure 8(a) plots the $V_{z} \propto z^{4}$ mode in the weak instability regime where $\Im(\hat{\lambda})=5<3 k_{\xi} \sigma_{z} / 4$. In this case the perturbation is approximately azimuthally symmetric as expected for an angular $m=0$ mode, but also has significant structure in the radial (action) direction. We therefore expect that this mode will be strongly damped by the 

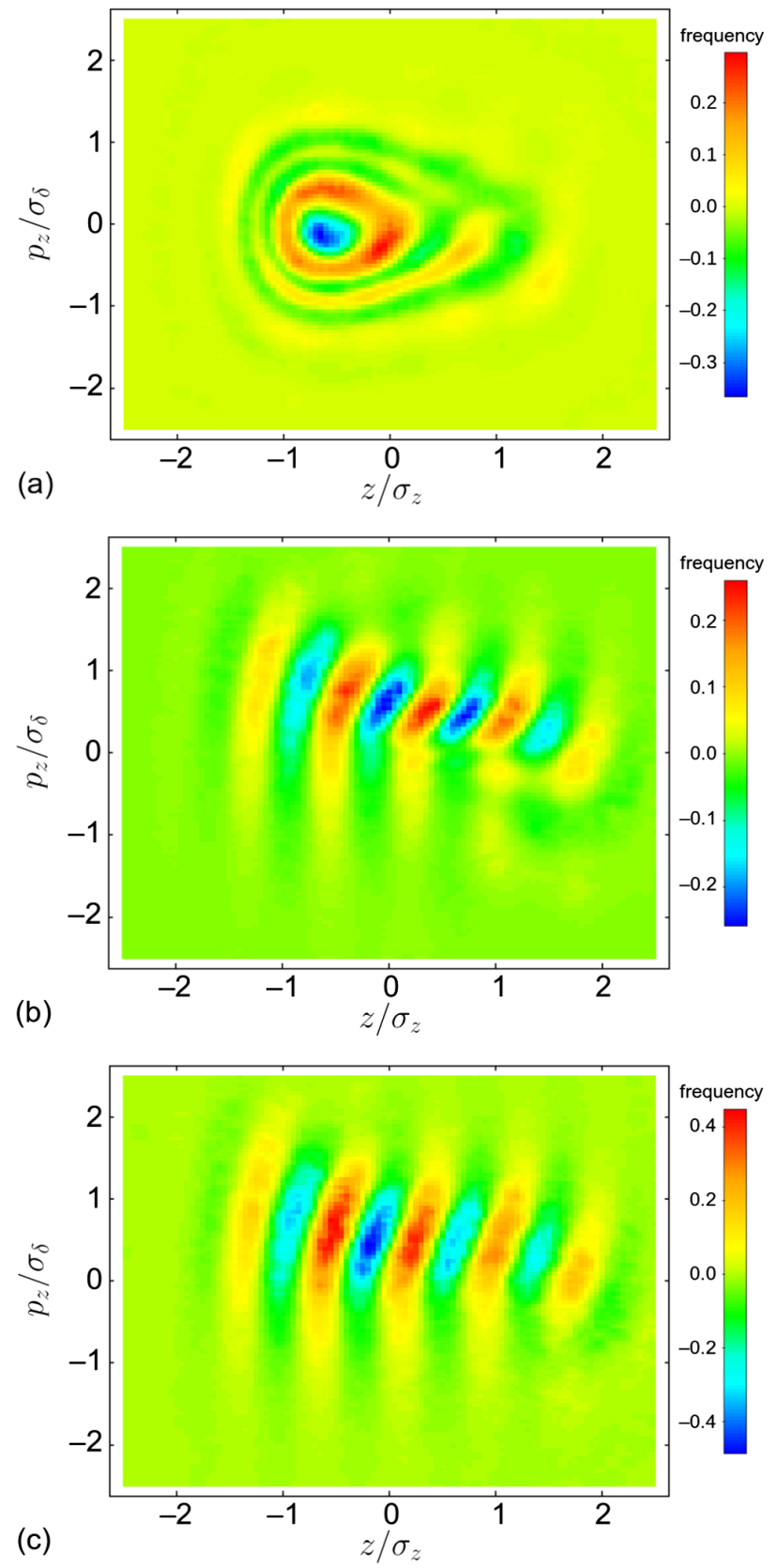

FIG. 8. Unstable longitudinal phase space density perturbations extracted from ELEGANT simulations for the quartic potential and $k_{\xi} \sigma_{z}=8$. Panel (a) has no synchrotron emission and shows that while the instability is dominated by the $m=0$ mode it has complicated radial dependence when $\Im(\hat{\lambda})=5$ and chromaticity plays an important role to control the instability. When the strength increases to $\hat{\lambda}=7>3 k_{\xi} \sigma_{z} / 4$ we get the results in panel (b), for which the perturbation becomes more aligned with the $p_{z}$ axis. Panel (c) is for the same parameters as panel (b) but also includes synchrotron emission.

diffusion of sychrotron emission, much like its quadratic potential counterpart.

On the other hand, Fig. 8(b) shows that when $\Im(\hat{\lambda})=$ $7>3 k_{\xi} \sigma_{z} / 4$ the unstable mode consists of stripes that

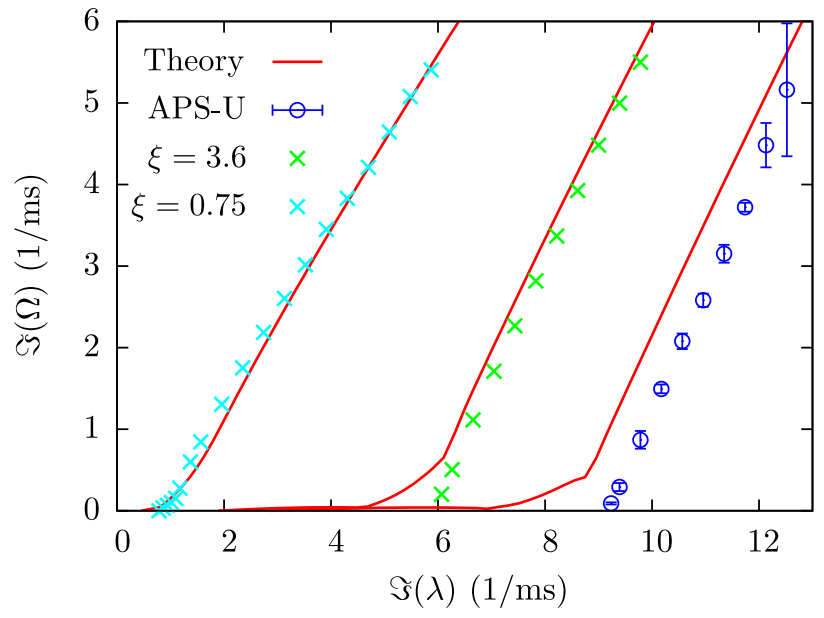

FIG. 9. Resistive wall growth rate as a function of $\xi=0$ rate $\lambda$ for the APS-U. The theory subtracts off the transverse damping rate $1 / \tau_{y} \approx 0.0651 / \mathrm{ms}$, while the tracking results include the nonlinear tune shift with amplitude and chromaticity; the APS-U (blue dots) have the nominal chromaticity $\xi_{y}=5.45$ for which $k_{\xi} \sigma_{z} \approx 12.1$. The green and points show results for $\xi_{y}=3.6$ and $\xi_{y}=0.75$, respectively; the latter is nominally unstable to the APS-U long-range resistive wall wakefield, whose growth rate $\Im(\lambda) \approx 0.881 / \mathrm{ms}$.

are nearly parallel to the $p_{z}$-axis, so that in this case diffusion would play little role. Hence, it is not surprising that panel (c) displays a very similar mode shape when synchrotron emission is included. Furthermore, both panels (b) and (c) look very similar to their quadratic counterparts in Fig. 5(b)-(c). This makes sense, because in the strong instability limit the growth rate is larger than the characteristic synchrotron frequency and the longitudinal potential does not significantly affect the dynamics.

As discussed previously, the physics of diffusion associated with synchrotron emission can stabilize the coupled bunch dynamics when $\Im(\lambda) \ll \xi \omega_{0} \sigma_{\delta}$, and this effect occurs regardless of the longitudinal potential. Indeed, including the diffusion due to synchrotron radiation for the APS-U essentially eliminates the weak instability in a manner similar to that observed in Fig. 3. We demonstrate this in Fig. 9, where we compare theoretical coupled-bunch growth rates from Eq. (32) to full ELEGANT simulations of the APS-U lattice. The red lines in Fig. 9 are the theory, while the blue points are the planned APS-U lattice with $\xi=5.45$. The simulation predicts that the APS-U will be stable to coupled-bunch instabilities whose $\xi=0$ growth rate $\Im(\lambda) \lesssim 9 \mathrm{~ms}^{-1}$. For comparison, applying Eqs. (E2) and (E7) to the APS-U vacuum chambers results in a ten times smaller long-range resistive wall $\Im(\lambda) \approx 0.9 \mathrm{~ms}^{-1}$, and the APS-U is stable by a safe margin. Figure 9 also includes the magenta and cyan points for illustration, with the former assuming a chromaticity of 2 , while the latter is marginally unstable at $\xi=0.75$. Note that neither of these are planned operating points, since $\xi=5.5$ was chosen to 
improve both the single-bunch collective stability and the single-particle dynamics of the APS-U hybrid lattice.

The simulations of Fig. 9 added both the diffusion due to synchrotron emission and a number of nonlinear effects, including the first and second order nonlinear tune shift with amplitude, the second and third order nonlinear chromaticities, the lowest order energy dependence of the lattice functions, and the lowest order variation of the path length with energy and amplitude. While adding nonlinear effects results in entirely different large-amplitude behavior including instability saturation and sawtooth-type behavior, Fig. 9 shows that adding nonlinearities does not significantly change the instability growth rates. The tune-shift with amplitude does not affect stability because the small $\varepsilon_{x}$ implies that the tune-shift over the bunch is negligible, while the nonlinear chromaticities shifts the growth rate curve by a few percent along $\Im(\lambda)$; for the APS-U adding the second order chromaticity increases the growth rates by shifting the curve left by $\sim 2.5 \%$ of $\Im(\lambda)$, while the third order chromaticity decreases $\Im(\Omega)$ slightly such that the curve shifts right by $\sim 0.5 \%$. These differences are relatively insignificant here, but they can lead to a sizeable fractional change in $\Im(\Omega)$ when $\Im(\hat{\Omega})<1$. If, for example, we artificially eliminate the diffusion we have found that nonlinear chromatic effects in the APS-U can increase the growth rates by up to $20 \%$; further analysis of this effect is beyond the scope of this paper.

\section{CONCLUSIONS}

We have derived a relatively easy-to-evaluate expression for computing the transverse coupled-bunch instability growth rates at any chromaticity and for both quadratic and quartic longitudinal potentials. Furthermore, we have generalized the former to include synchrotron emission, and shown that this has an important stabilizing effect at large chromaticity. The theory predicts that the coupledbunch growth rates can be significantly reduced when the chromatic betatron tune spread is larger than the coupledbunch growth rate at zero chromaticity, and was shown to agree quite well with tracking simulations for quadratic and quartic (flattened) longitudinal potentials.

\section{ACKNOWLEDGMENTS}

We would like to thank our APS colleagues M. Borland, J. Calvey, and L. Emery for useful comments. We would also like to recognize our discussions with G. Bassi, A. Blednykh, and M. Venturini. This work was supported by U.S. Dept. of Energy Office of Sciences under Contract No. DE-AC02-06CH11357.

\section{APPENDIX A: EXTENSION TO CERTAIN NONUNIFORM FILLS}

This calculation is very similar to that in Ref. [33], but corrects a few typos. We let $T_{0} / M$ be the minimum spacing between bunches (equispaced bunches have $M=N_{b}$ ), and abbreviate the dispersionlike integration over the equilibrium $\bar{f}_{n}$ that is in Eqs. (25) and (30):

$$
\begin{aligned}
\mathcal{X}_{n}(\Omega)= & \int_{0}^{\infty} d \mathcal{I} \frac{2 \pi \bar{f}_{n}(\mathcal{I})}{\omega(\mathcal{I})\left(1-e^{2 \pi i \Omega}\right)} \\
& \times \int_{0}^{2 \pi} d \theta J_{0}\left[2 k_{\xi} \zeta(\mathcal{I}) \sin (\theta / 2)\right] e^{i \Omega \theta / \omega(\mathcal{I})} .
\end{aligned}
$$

Then, from Eqs. (26) and (27) we find that stability is determined by the solutions to

$$
\frac{\mathcal{D}_{n}}{\mathcal{X}_{n}}=\sum_{j=0}^{M-1} \frac{e^{2} N_{e, j}}{2 \gamma m c^{2} T_{0}} \mathcal{D}_{j} \sum_{\ell=0}^{\infty} e^{i \ell\left(\omega_{\beta}+\omega_{Q}\right) T_{0}} W_{D}(\Upsilon),
$$

where $\Upsilon=-c T_{0}[\ell-(j-n) / M]$. To make further progress we introduce the coupled-bunch modes

$$
\tau_{\mu}=\sum_{n=0}^{M-1} \frac{\mathcal{D}_{n}}{\mathcal{X}_{n}} e^{-2 \pi i n \mu / M} e^{-i n\left(\omega_{\beta}+\omega_{Q}\right) T_{0} / M},
$$

in terms of which (A2) can be written as

$$
\begin{aligned}
\tau_{\mu} & =\sum_{j, \mu^{\prime}=0}^{M-1} \frac{e^{2} N_{e, j} \mathcal{X}_{j}}{2 \gamma m c^{2} T_{0}} e^{2 \pi i j\left(\mu^{\prime}-\mu\right) / M} \tau_{\mu^{\prime}} \sum_{n=0}^{M-1} \sum_{\ell=0}^{\infty} e^{-i\left(\omega_{\beta}+\omega_{Q}+2 \pi \mu / T_{0}\right) \Upsilon / c} W_{D}(\Upsilon) \\
& =\sum_{j, \mu^{\prime}=0}^{M-1} \frac{e^{2} N_{e, j} \mathcal{X}_{j}}{2 \gamma m c^{2} T_{0}} e^{2 \pi i j\left(\mu^{\prime}-\mu\right) / M} \tau_{\mu^{\prime}} \sum_{k=0}^{\infty} e^{i\left[\left(\omega_{\beta}+\omega_{Q}\right) T_{0}+2 \pi \mu\right] k / M} W_{D}\left(-k c T_{0} / M\right) .
\end{aligned}
$$

The first line follows from inserting the definition (A3) into both sides of (A2), multiplying by $e^{2 \pi i n \mu / M} e^{i n\left(\omega_{\beta}+\omega_{Q}\right) T_{0} / M}$ and summing over $n$, and using the fact that $e^{2 \pi i \mu \ell}=1$ for integer $\mu, \ell$; in the second line we have exchanged the summation over $n$ and $\ell$ to a single sum over $k$ as was suggested in Ref. [36]. Continuing their analysis we then separate the summation over $\mu^{\prime}$ to one where $\mu^{\prime}=\mu$ and the rest to arrive at 


$$
\begin{aligned}
\tau_{\mu}= & \tau_{\mu} \sum_{j=0}^{M-1} \frac{e^{2} N_{e, j} \mathcal{X}_{j}}{2 \gamma m c^{2} T_{0}} \sum_{k=0}^{\infty} e^{i\left[\left(\omega_{\beta}+\omega_{Q}\right) T_{0}+2 \pi \mu\right] k / M} W_{D}\left(-k c T_{0} / M\right) \\
& +\sum_{j=0}^{M-1} \sum_{\mu^{\prime} \neq \mu}^{M-1} \frac{e^{2} N_{e, j} \mathcal{X}_{j}}{2 \gamma m c^{2} T_{0}} e^{2 \pi i j\left(\mu^{\prime}-\mu\right) / M} \tau_{\mu^{\prime}} \sum_{k=0}^{\infty} e^{i\left[\left(\omega_{\beta}+\omega_{Q}\right) T_{0}+2 \pi \mu\right] k / M} W_{D}\left(-k c T_{0} / M\right) .
\end{aligned}
$$

The first term represents the diagonal part of the matrix, while the second term gives the coupling between modes $\tau$. For uniformly spaced identical bunches the second line vanishes and each term in the first sum over $j$ is the same. This is the standard case. In addition, the second coupling term in (A5) also vanishes if the bunch pattern is comprised of two or more approximately identical bunch trains; in this case stability is given by setting the weighted sum of dispersion integrals from the top lines of (A5) equal to unity.

\section{APPENDIX B: GREEN FUNCTION SOLUTION OF THE APPROXIMATE FOKKER-PLANCK EQUATION}

In this Appendix we derive the Green function $\mathcal{G}(r, \hat{r})$ associated with the differential operator on the left-hand side of our simplified diffusion equation (50), and then use $\mathcal{G}$ to solve for the mode coefficient $g_{n}^{m}$. To begin, we note that the spectral theorem states that a self-adjoint operator has a complete set of eigenfunctions $\Psi_{p}(r)$ with eigenvalues $\Lambda_{p}$, from which the associated Green function can then be constructed using

$$
\mathcal{G}(r, \hat{r})=\sum_{p=0}^{\infty} \frac{1}{\Lambda_{p}} \Psi_{p}^{\dagger}(r) \Psi_{p}(\hat{r})
$$

Additionally, we can convert the left-hand side of (50) into a self-adjoint form by multiplying by $e^{r / 2}$ and using the fact that

$$
\begin{aligned}
& e^{r / 2}\left[r \frac{d^{2}}{d r^{2}}+(1+r) \frac{d}{d r}-\frac{m^{2}}{4 r}+1+\frac{i \tau_{z}}{2}\left(\Omega-m \omega_{s}\right)-\frac{\tau_{z}}{2 \tau_{y}}\right] g_{n}^{m}=\mathscr{L}\left[e^{r / 2} g_{n}^{m}\right] \\
= & {\left[r \frac{d^{2}}{d r^{2}}+r \frac{d}{d r}+\frac{r}{4}-\frac{m^{2}}{4 r}+\frac{1}{2}+\frac{i \tau_{z}}{2}\left(\Omega-m \omega_{s}\right)-\frac{\tau_{z}}{\tau_{y}}\right]\left(e^{r / 2} g_{n}^{m}\right)=i \tau_{z} \frac{e^{-r / 2} J_{m}\left(k_{\xi} \sigma_{z} \sqrt{2 r}\right)}{4 \pi i^{m} \sigma_{z} \sigma_{\delta}} \sum_{j=0}^{N_{b}-1} \mathrm{M}_{n, j} \mathcal{D}_{j}(\Omega), }
\end{aligned}
$$

where we defined the self-adjoint linear operator $\mathscr{L}$. This operator satisfies $\mathscr{L}\left[\Psi_{p}(r)\right]=\Lambda_{p} \Psi_{p}(r)$ with orthonormal eigenfunctions $\Psi_{p}(r)=r^{|m / 2|} L_{p}^{|m|}(r) e^{-r / 2}[p ! /(p+|m|) !]$ and eigenvalues $\Lambda_{p}=i\left[\tau_{z}\left(\Omega-m \omega_{s}\right)+i \tau_{z} / \tau_{y}+i(2 p+|m|)\right]$, which when inserted into Eq. (B1) yields the Green function

$$
\mathcal{G}_{m}(r, \hat{r})=\frac{2}{i \tau_{z}} \sum_{p} \frac{p ! r^{|m / 2|} L_{p}^{|m|}(r) e^{-r / 2} \hat{r}^{|m / 2|} L_{p}^{|m|}(\hat{r}) e^{-\hat{r} / 2}}{(p+|m|) !\left[\Omega-m \omega_{s}+i / \tau_{y}+i(2 p+|m|) / \tau_{z}\right]} .
$$

We now use the Green function (B3) to invert (B2) and the following solution for $g_{n}^{m}$ as given in Eq. (51).

$$
\begin{aligned}
g_{n}^{m}(r) & =e^{-r / 2} \int_{0}^{\infty} d \hat{r} \mathcal{G}_{m}(r, \hat{r}) i \tau_{z} \frac{e^{-\hat{r} / 2} J_{m}\left(k_{\xi} \sigma_{z} \sqrt{2 \hat{r}}\right)}{4 \pi i^{m} \sigma_{z} \sigma_{\delta}} \sum_{j=0}^{N_{b}-1} \mathrm{M}_{n, j} \mathcal{D}_{j}(\Omega) \\
& =\sum_{p=0}^{\infty} \frac{\left(k_{\xi} \sigma_{2} / \sqrt{2}\right)^{2 p+|m|} e^{k_{\xi}^{2} \sigma_{z}^{2} / 2}}{\Omega-m \omega_{s}+i / \tau_{y}+i(2 p+|m|) / \tau_{z}} \frac{e^{-r} r^{|m / 2|} L_{p}^{|m|}(r)}{2 \pi i^{m} \sigma_{z} \sigma_{\delta}(p+|m|) !} \sum_{j=0}^{N_{b}-1} \mathrm{M}_{n, j} \mathcal{D}_{j}(\Omega)
\end{aligned}
$$

\section{APPENDIX C: ALTERNATE INTEGRAL SOLUTION FOR VLASOV STABILITY}

In this Appendix we derive another integral equation for $\Omega$ that we have found is more efficient to numerically solve than Eq. (32) when the potential is not harmonic. To obtain this alternative expression, we begin with Eq. (28). This time, we 
insert $z(\mathcal{I}, \Phi)=\zeta(\mathcal{I}) \cos \Phi$ and perform the angular integrations using the Jacobi-Anger identity; going step by step, the right-hand side of (28) becomes

$$
\begin{aligned}
\int_{0}^{2 \pi} d \Phi \frac{e^{i k_{\xi} z(\Phi, \mathcal{I})}}{2 \pi i \omega(\mathcal{I})} \int_{0}^{2 \pi} d \theta \frac{e^{i \Omega \theta / \omega}}{1-e^{2 \pi i \Omega / \omega}} e^{-i k_{\xi} \zeta(\mathcal{I}) \cos (\Phi-\theta)} & =\int_{0}^{2 \pi} d \Phi \frac{e^{i k_{\xi} z(\Phi, \mathcal{I})}}{2 \pi i \omega(\mathcal{I})} \int_{0}^{2 \pi} d \theta \sum_{n=-\infty}^{\infty} \frac{e^{i \Omega \theta / \omega} e^{i n(\Phi-\theta)}}{i^{n}\left(1-e^{2 \pi i \Omega / \omega}\right)} J_{n}\left(k_{\xi} \zeta\right) \\
& =\int_{0}^{2 \pi} d \Phi \sum_{m=-\infty}^{\infty} i^{m} J_{m}\left(k_{\xi} \zeta\right) \frac{e^{i m \Phi}}{2 \pi} \sum_{n=\infty}^{\infty} \frac{i^{n} J_{-n}\left(k_{\xi} \zeta\right) e^{i n \Phi}}{\Omega-n \omega(\mathcal{I})} \\
& =\sum_{m=-\infty}^{\infty} \frac{J_{m}\left[k_{\xi} \zeta(\mathcal{I})\right]^{2}}{\Omega-m \omega(\mathcal{I})} .
\end{aligned}
$$

Then, inserting (C1) into (28) implies that stability is given by the dimensionless equation

$$
1=\hat{\lambda} \int_{0}^{\infty} d \mathcal{I} 2 \pi \bar{f}(\mathcal{I}) \sum_{m=-\infty}^{\infty} \frac{J_{m}\left[k_{\xi} \zeta(\mathcal{I})\right]^{2}}{\hat{\Omega}-m \hat{\omega}(\mathcal{I})} .
$$

We find that Eq. (C2) has traded one integral from (32) at the cost of an infinite sum. The number of terms required for the sum to converge increases linearly with $k_{\xi} \sigma_{z}$, and we have found that restricting the sum to $|m| \leq 25$ is sufficient for $k_{\xi} \sigma_{z}=8$. Finally, when the potential is simple harmonic we can insert (36) into Eq. (C2) and integrate to get the following expression in terms of the modified Bessel function $I_{n}(x)$ :

$$
1=\hat{\lambda} \sum_{m=-\infty}^{\infty} \frac{I_{m}\left(k_{\xi}^{2} \sigma_{z}^{2}\right)}{\hat{\Omega}-m} e^{-k_{\xi}^{2} \sigma_{z}^{2}} .
$$

\section{APPENDIX D: INSTABILITY THEORY FOR A "WATERBAG" LONGITUDINAL EQUILIBRIUM}

All previous results in this paper assume that the unperturbed momentum distribution is Gaussian, so that $\bar{f}(\mathcal{I}) \propto e^{-\mathcal{I} / \sigma_{z} \sigma_{\delta}}$. While this is appropriate when the equilibrium is dictated by synchrotron radiation, other functions may better describe the beam when radiation damping is negligible. Our formalism accounts for the longitudinal equilibrium $\bar{f}$ as indicated by (C2), and in this Appendix we illustrate to what extent a different $\bar{f}$ might change previous predictions. We do this by applying the simple harmonic theory to the so-called "waterbag" distribution, which is constant within a specified phase space ellipse and zero elsewhere:

$$
2 \pi \bar{f}(\mathcal{I})=\left\{\begin{array}{ll}
\left(2 \sigma_{z} \sigma_{\delta}\right)^{-1} & \text { if } \mathcal{I} \leq 2 \sigma_{z} \sigma_{\delta} \\
0 & \text { if } \mathcal{I}>2 \sigma_{z} \sigma_{\delta}
\end{array} .\right.
$$

Analytic results can also be computed for, e.g., air-bag or parabolic models, but we think that the waterbag distribution exemplifies how predictions depend on the equilibrium, while also balancing realism with simplicity. Inserting $\bar{f}$ from (D1) into (C2) and integrating yields [28]

$$
1=\hat{\lambda} \sum_{m=-\infty}^{\infty} \frac{J_{m}^{2}(a)-J_{m+1}(a) J_{m-1}(a)}{\hat{\Omega}-m},
$$

where $a=2 k_{\xi} \sigma_{z}$.

We compare the predictions for the instability growth rate for a waterbag distribution derived from Eq. (D2) with the Gaussian theory of (C3) in Fig. 10. Figure 10 plots the maximum theoretical growth rate for $k_{\xi} \sigma_{z}=4$ and $k_{\xi} \sigma_{z}=4$, and compares these results with those derived from simulation. We see that all cases show very similar behavior, and in particular demonstrate the same characteristic weak and strong instability regimes that arise when $k_{\xi} \sigma_{z}$ is less than or greater than $3 \Im(\hat{\jmath})$, respectively. Furthermore, the chromatic stabilizing effect is somewhat stronger for the waterbag distribution than for the Gaussian with a same value of $\sigma_{z}$, and these differences are well predicted by the theory.

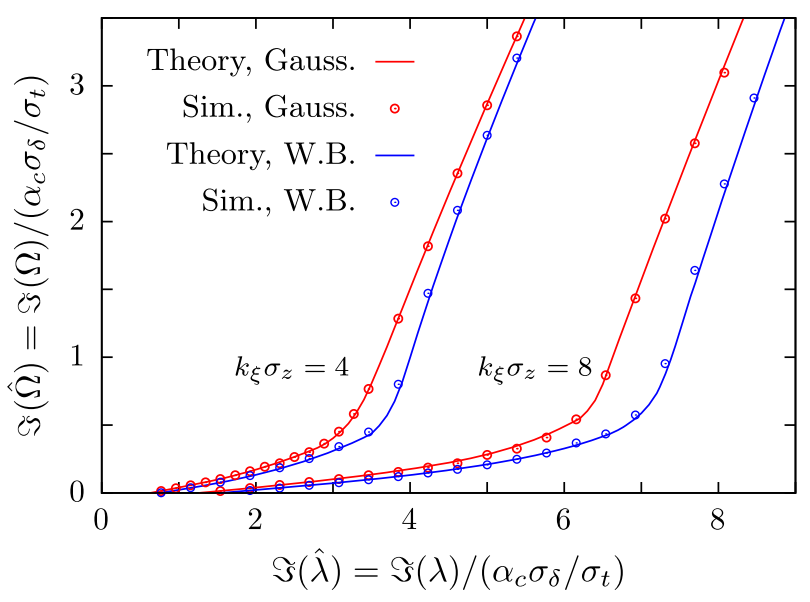

FIG. 10. Comparison of the maximum growth rate for Gaussian (Gauss.) and waterbag (W.B.) equilibriums, assuming that the longitudinal potential is simple harmonic. 


\section{APPENDIX E: LONG-RANGE RESISTIVE WALL INSTABILITY}

Here we compute the $\xi=0$ complex frequencies $\lambda_{\mu}$ for the long-range resistive wall instability. Although most of the elements of this calculation can be found by combining references such as [1] and [37], we were unable to find the desired approximation for $\lambda$ suitable to the parameters of Table I. We will derive it from Eq. (A5), starting with the fact that at zero chromaticity Eq. (A1) implies that $\chi_{n}=1 / \Omega=1 / \lambda$. We furthermore assume that the quadrupolar frequency shift $\omega_{Q}=0$ and that there are $M=N_{b}$ equally spaced bunches with equal charge $N_{e, j}=N_{e}$. In this case the second summation in (A5) vanishes, while the first sum is over $N_{b}$ identical terms so that the eigenvalues are given by

$$
\lambda_{\mu}=\frac{e I_{\text {tot }}}{2 \gamma m c^{2} T_{0}} \sum_{k=0}^{\infty} e^{2 \pi i\left(\mu+\nu_{\beta}\right) k / M} W_{D}\left(-k c T_{0} / M\right) .
$$

Here, we have defined the vertical tune $\nu_{\beta}=\omega_{\beta} T_{0} / 2 \pi$ and the total current $I_{\text {tot }}=e N_{e} N_{b} / T_{0}$.

The long-range resistive-wall wakefield is zero when $z \geq 0$, and otherwise scales as $|z|^{-1 / 2}$ with the prefactor determined by summing up the contributions from various chambers around the ring. Labeling each chamber type with the subscript $\ell$, the long-range resistive wall wakefield can be written as [1]

$$
W_{D}(z)=\sum_{\ell} \frac{g_{\ell} \beta_{y, \ell}}{\pi b_{\ell}^{3}} \sqrt{\frac{Z_{0} \rho_{\ell}}{\pi}} \frac{c L_{\ell}}{\sqrt{-z}}=\mathcal{W}_{y} \sqrt{\frac{c T_{0}}{-z}},
$$

where $\beta_{y, \ell}, b_{\ell}, \rho_{\ell}$, and $L_{\ell}$ are, respectively, the average beta function, vertical aperture, resistivity, and length of chamber $\ell$, while $g_{\ell}$ is a geometric factor that is unity for round chambers and $\pi^{2} / 12$ for the highly elliptical insertion device chambers used by the APS-U. Inserting the ring-averaged resistive wall wakefield (E2) into (E1) implies that the eigenvalues are

$$
\begin{aligned}
\lambda_{\mu} & =-\frac{e I_{\text {tot }}}{2 \gamma m c^{2}} \frac{\mathcal{W}_{y}}{\sqrt{N_{b}}} \sum_{k=1}^{\infty} \frac{e^{2 \pi i\left(\mu+\nu_{\beta}\right) k / N_{b}}}{\sqrt{k}} \\
& =-\frac{e^{2} I_{\mathrm{tot}}}{2 \gamma m c^{2}} \frac{\mathcal{W}_{y}}{\sqrt{N_{b}}} \operatorname{Li}_{\frac{1}{2}}\left[e^{2 \pi i\left(\mu+\nu_{\beta}\right) / N_{b}}\right],
\end{aligned}
$$

where $\mathrm{Li}_{1 / 2}(x)$ is the polylogarithm function [35]. From (E3) we see that the summation can be large when $(\mu+$ $\left.\nu_{\beta}\right) / N_{b}$ is small. In this case we apply the Taylor expansion

$$
\mathrm{Li}_{1 / 2}\left(e^{2 \pi i x}\right)=\frac{1+i}{2 \sqrt{x}}+\zeta(1 / 2)+O(x)
$$

for $|x| \ll 1$ and the Riemann zeta function $\zeta(1 / 2) \approx-1.46$. Then, we find that the growth rate is maximized for the mode number $\mu$ that has $\mu+\nu_{\beta}=-1+\left\{\nu_{\beta}\right\}$, where $\left\{\nu_{\beta}\right\}$ is the fractional tune. The complex coupled-bunch frequency with largest growth rate is therefore

$$
\lambda \approx \frac{e I_{\text {tot }} \mathcal{W}_{y}}{4 \gamma m c^{2} \sqrt{1-\left\{\nu_{\beta}\right\}}}\left[i-\left(1+2 \zeta(1 / 2) \sqrt{\frac{1-\left\{\nu_{\beta}\right\}}{N_{b}}}\right)\right] .
$$

The correction to the real part involving $\zeta(1 / 2)$ is typically neglected, since it does not change the $\xi=0$ growth rate. It does, however, affect both the real and imaginary parts of $\Omega$ at nonzero chromaticity, and for the APS-U parameters it is an important factor. In particular, when $N_{b}=48$ and $\left\{\nu_{\beta}\right\}=0.1$ we find that

$$
\lambda \approx \frac{e I_{\text {tot }} \mathcal{W}_{y}}{4 \gamma m c^{2} \sqrt{1-\left\{\nu_{\beta}\right\}}}(i-0.6)=\Im(\lambda)(i-0.6) .
$$

Hence, for APS-U the lowest order correction involving $\zeta(1 / 2)$ changes $\Re(\lambda)$ by $40 \%$; the neglected higher-order terms in (E5) change $\lambda$ by less than $1 \%$.

\section{APPENDIX F: DETAILS OF THE ELEGANT SIMULATIONS}

We track particles through the ring using the ILMATRIX element in ELEGANT, which allows for fast, symplectic particle tracking through a periodic cell including chromatic and amplitude-dependent tunes, beta functions, dispersion, and path-length. ILMATRIX does this by computing a linear matrix for each particle that is determined both by user-supplied parameters and the particle coordinates. The user-supplied quantities include the Twiss parameters, tunes, dispersion, etc., and how these quantities depend on the particle energy (giving chromatic effects through third order in $p_{z}$ ) and on the transverse coordinates (which provide the nonlinear terms in $\mathcal{J}$ ). We have found that the ILMATRIX element provides a fast and reasonably accurate way to track particles through a wide variety of lattices. For all simulations reported here other than those in Fig. 9 we only include the linear and chromatic terms; we have found numerically that setting the second and third order chromatic effects to zero only changes the growth rates by $\lesssim 15 \%$ in the weak regime, and $\lesssim 5 \%$ when the instability is strong. On the other hand, including the other nonlinear dependences does not affect the instability thresholds, but does change the large amplitude oscillation dynamics. In other words, for our parameters the beam emittances are sufficiently small that stability is determined predominantly by the linear lattice and chromaticity, while the nonlinear chromaticities provides a small correction to that.

The multiple bunches are initialized from a single equilibrium of 50,000 particles that is copied 47 times 
and assigned evenly about the ring to arrive at the total of 48 bunches. The number of macroparticles required increases with chromaticity and when the rf potential is not harmonic. We found that increasing the number of particles to 200,000 gave nearly indistiguishable results for the quartic potential at the highest value of $\xi$, while decreasing the number to 10,000 gave good results for most cases except at the highest chromaticity in the quartic potential; for consistency we decided to use 50,000 particles for all cases. We simulate the distributed longrange resistive wall wakefield using one LRWAKE element every other sector for a total of 20 elements per turn; using multiple LRWAKE elements is only truly required when the $\xi=0$ growth rate becomes large. We apply the resistive wall wake summed over 25 turns, where the first 20 turns are given by the analytic expression Eq. (E2), after which the wakefield is smoothly brought to zero over the final five turns using a Gaussian envelope. This ensures that the numerical sum approximates its infinite value from Eq. (E3) to better than $0.2 \%$. We have also simulated a HOM driven wakefield using the RFMODE element, for which the wakefield is computed using the fundamental theorem of beam loading and a phasor rotation. While the LRWAKE element uses a point bunch approximation for the wakefield, RFMODE does not, and we have seen no discrepancies from the theory in either case.

The acceleration and focusing from the rf cavity (or cavities) is modeled using the RFCA element(s), which simulate active cavities with a prescribed frequency, phase, and voltage. We complete our tracking simulation by modeling the damping and diffusion due to synchrotron emission using the SREFFECTS element, which takes as input the energy loss per turn, damping partitions, and equilibrium emittances and energy spread.

Finally, we obtained the perturbed distribution Figs. 5 and 8 from the ELEGANT simulation by making a weighted histogram of the $N_{\text {sim }}$ macroparticles in a bunch, where the weight on the $j^{\text {th }}$ particle is

$$
\frac{y_{j}+\beta_{y} y_{j}^{\prime}}{\langle y\rangle} \frac{e^{i\left(\omega_{\beta} s / c-k_{\xi} z_{j}\right)}}{N_{\operatorname{sim}}(\Delta z)\left(\Delta p_{z}\right)},
$$

with $\Delta z$ and $\Delta p_{z}$ the bin size in $z$ and $p_{z}$ respectively, and the division by the centroid amplitude $\langle y\rangle$ is done to normalize the data near unity. The plots in Figs. 5 and 8 show the real part of (F1) as a function of $z$ and $p_{z}$ (the imaginary parts are similar), and use a nearest neighbor smoothing to reduce granularity due to a finite number of macroparticles.

[1] A. W. Chao, Physics of Collective Beam Instabilities in High Energy Accelerators (Wiley, New York, 1993).

[2] K. Y. Ng, Physics of Intensity Dependent Beam Instabilities (World Scientific, Singapore, 2006).
[3] E. D. Courant and A.M. Sessler, Transverse coherent resistive instabilities of azimuthally bunched beams in particle accelerators, Rev. Sci. Instrum. 37, 1579 (1966).

[4] K. A. Thompson and R. D. Ruth, Transverse and longitudinal coupled-bunch instabilities in trains of closely spaced bunches, in Proc. of 1989 Particle Accel. Conf., Chicago, IL, 1989 (IEEE, New York, 1989), page 792. SLAC-PUB-4872.

[5] F. Sacherer, Methods for computing bunched-beam instabilities, CERN/SI-BR Report No. 72-5, 1972.

[6] F. Sacherer, Transverse bunched-beam instabilities - theory, in Proc. 9th Int. Conf. on High Energy Accelerators, Washington, DC (SLAC, Stanford, CA, 1974), p. 347.

[7] B. Zotter and F. Sacherer, Transverse instabilities of relativistic particle beams in accelerators and storage rings, in Theoretical Aspects of the Behaviour of Beams in Accelerators and Storage Rings (CERN, Geneva, 1976), p. 198.

[8] B. Zotter, Sacherer formulae, in Handbook of Accelerator Physics and Engineering, edited by A. W. Chao and M. Tigner (World Scientific, Singapore, 2013), p. 120.

[9] J. L. Laclare, Bunched beam coherent instabilities, in CERN Accelerator School: Advanced Accelerator Physics (CERN, Geneva, 1985), p. 264.

[10] F. J. Cullinan, R. Nagaoka, G. Skripka, and P. F. Tavares, Transverse coupled-bunch instability thresholds in the presence of a harmonic-cavity-flattened rf potential, Phys. Rev. Accel. Beams 19, 124401 (2016).

[11] M. Borland, ELEGANT: A flexible sdds-compliant code for accelerator simulation, Advanced Light Source Technical Report No. LS-287, 2000.

[12] R. Warnock, M. Venturini, and J. A. Ellison, Nonsingular integral equation for stability of a bunched beam, in Proceedings of the 8th European Particle Accelerator Conference, Paris, 2002 (EPS-IGA and CERN, Geneva, 2002), p. 1589.

[13] R. L. Warnock, G. V. Stupakov, M. Venturini, and J. A. Ellison, Linear Vlasov analysis for stability of a bunched beam, in Proceedings of the 9th European Particle Accelerator Conference, Lucerne, 2004 (EPS-AG, Lucerne, 2004), p. 2215 [http://accelconf.web.cern.ch/AccelConf/e04/].

[14] M. Venturini, Harmonic cavities and the transverse modecoupling instability driven by a resistive wall, Phys. Rev. Accel. Beams 21, 024402 (2018).

[15] J. M. Wang and C. Pelligrini, On the condition for a single bunch high frequency fast blow-up, in Proc. XI Int. Conf. on High Energy Accel. (CERN, Geneva, Switzerland, 1980), p. 554, BNL-28034.

[16] R. D. Ruth and J. M. Wang, Vertical fast blow-up in a single bunch, IEEE Trans. Nucl. Sci. 28, 2405 (1981).

[17] S. Krinsky and J. M. Wang, Longitudinal instabilities of bunched beams subject to a non-harmonic rf potential, Part. Accel. 17, 109 (1985).

[18] A. Burov, Coupled-beam and coupled-bunch instabilities, Phys. Rev. Accel. Beams 21, 114401 (2018).

[19] H. Goldstein, Classical Mechanics, 2nd ed. (AddisonWesley, Reading, MA, 1980).

[20] I. Percival and D. Richards, Introduction to Dynamics (Cambridge University Press, Cambridge, United Kingdom, 1982). 
[21] C. Pellegrini, On a new instability in electron-positron storage rings (the head-tail effect), Nuovo Cimento 64, 447 (1969).

[22] M. Sands, The head-tail effect: an instability mechanism in storage rings, SLAC Report No. 8, 1969.

[23] A. Chao, S. Heifets, and B. Zotter, Tune shifts of bunch trains due to resistive vacuum chambers without circular symmetry, Phys. Rev. Accel. Beams 5, 111001 (2002).

[24] H. Wiedemann, Particle Accelerator Physics I (SpringerVerlag, Berlin, 1993).

[25] T. Suzuki, Fokker-Planck theory of transverse modecoupling instability, Part. Accel. 20, 79 (1986).

[26] T. Nakamura, K. Soutome, M. Takao, S. Takano, T. Ohshima, M. Masaki, S. Sasaki, M. Shoji, and K. Tsumaki, Chromaticity for energy spread measurement and for cure of transverse multi-bunch instability in the SPring-8 storage ring, in Proceedings of the 19th Particle Accelerator Conference, Chicago, IL, 2001 (IEEE, Piscataway, NJ, 2001), p. 1972.

[27] R. R. Lindberg, Fokker-Planck analysis of transverse collective instabilities in electron storage rings, Phys. Rev. Accel. Beams 19, 124402 (2016).

[28] I. S. Gradshteyn and I. M. Ryzhik, Table of Integrals, Series, and Products, 6th ed. (Academic Press, New York, 2000).

[29] A. Andersson, P. F. Tavares, A. Hansson, and J. Breunlin, Equilibrium bunch density distribution with passive harmonic cavities in a storage ring, Phys. Rev. Accel. Beams 17, 064401 (2014).

[30] P. F. Tavares, E. Al-Dmour, A. Andersson, J. Breunlin, F. Cullinan, E. Mansten, S. Molloy, D. Olsson, D. K. Olsson, M. Sjöström, and S. Thorin, Status of the MAX IV accelerators, in Proc. 10th Int. Particle Accel. Conf. (JACoW, Geneva, 2019), p. 1185.

[31] APS Upgrade project final design review report - 2019. https://www.aps.anl.gov/APS-Upgrade/Documents.

[32] C. Steier et al., Design progress of ALS-U, the soft x-ray diffraction limited upgrade of the Advanced Light Source, In Proc. 10th Int. Particle Accel. Conf. (JACoW, Geneva, 2019), p. 1639.

[33] R. R. Lindberg, Theory of coupled-bunch longitudinal instabilities in a storage ring for arbitrary rf potentials, Phys. Rev. Accel. Beams 21, 124402 (2018).

[34] L. Landau, On the vibrations of the electronic plasma, J. Physics (USSR) 10, 25 (1946).

[35] Wolfram Research, Inc. Mathematica, Version 11.3. Champaign, IL, 2018.

[36] G. Bassi, A. Blednykh, and V. Smaluk, Self-consistent simulations and analysis of the coupled-bunch instability for arbitrary multibunch configurations, Phys. Rev. Accel. Beams 19, 024401 (2016).

[37] K. Bane, Y. Cai, A. Chao, R. Hettel, Z. Huang, Y. Nosochkov, G. Stupakov, L. Wang, and M.-H. Wang, Lattice and collective effects for PEP-X, SLAC Technical Report No. SLAC-PUB-13225, 2008. 